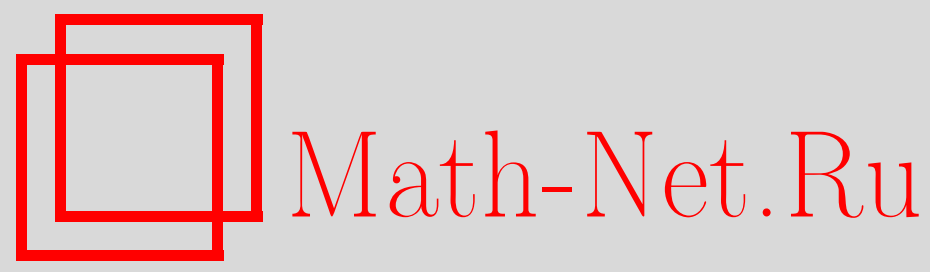

В. П. Маслов, О. Ю. Шведов, Об асимптотике матрицы плотности системы большого числа тождественных частиц, Матем. заметки, 1999, том 65, выпуск 1, 84-106

DOI: https://doi.org/10.4213/mzm1031

Использование Общероссийского математического портала Math-Net.Ru подразумевает, что вы прочитали и согласны с пользовательским соглашением http://www.mathnet.ru/rus/agreement

Параметры загрузки:

IP: 54.198 .55 .26

26 апреля 2023 г., 16:21:05

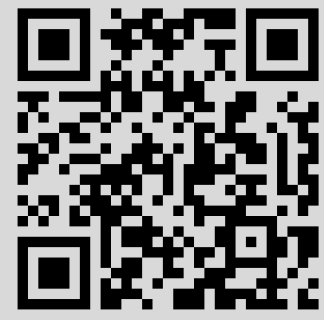




\title{
ОБ АСИМПТОТИКЕ МАТРИЦЫ ПЛОТНОСТИ СИСТЕМЫ БОЛЬШОГО ЧИСЛА ТОЖДЕСТВЕННЫХ ЧАСТИЦ
}

\author{
В.П. Маслов, О. Ю. Шведов
}

Рассматривается уравнение Вигнера для системы большого числа $N$ тождественных частиц, коэффициент взаимодействия между которыми порядка $1 / N$. Как в бозонном, так и в фермионном случаях строится асимптотика решения задачи Коши для этого уравнения с учетом обменных эффектов в случае, если постоянная Планка порядка $N^{-1 / d}$, где $d$ - размерность пространства. Данная асимптотика интерпретируется в терминах теории комплексного ростка на искривленном фазовом пространстве, которое эквивалентно пространству функций со значениями на римановой сфере в ферми-случае и на плоскости Лобачевского в бозе-случае. Классические уравнения движения в обоих случаях сводятся к уравнению Власова, однако из-за бесконечномерности фазового пространства на комплексный росток накладьваются дополнительные условия, зависящие от типа статистики.

Библиография: 12 названий.

1. Введение. В работе [1] рассмотрено построение при $N \rightarrow \infty$ приближенных решений уравнения Вигнера

$$
i \hbar \frac{d}{d t} \hat{\rho}_{N}^{t}=\left[\widehat{H}_{N}, \hat{\rho}_{N}^{t}\right]
$$

для зависящего от вещественного параметра $t$ эрмитова положительно определенного оператора $\hat{\rho}_{N}^{t}$ со сходящимся следом, действующего в пространстве $L^{2}\left(\mathbb{R}^{d N}\right), d \in \mathbb{N}$, функций $N$ аргументов $x_{1}, \ldots, x_{N} \in \mathbb{R}^{d}$. Оператор $\widehat{H}_{N}$, входящий в уравнение $(1)$, имеет вид

$$
\widehat{H}_{N}=\sum_{i=1}^{N}\left(-\frac{\hbar^{2}}{2} \frac{\partial^{2}}{\partial x_{i}^{2}}+U\left(x_{i}\right)\right)+\varepsilon \sum_{1 \leqslant i<j \leqslant N} V\left(x_{i}, x_{j}\right),
$$

где $\varepsilon=O(1 / N), U$ и $V$ - гладкие функции, $x_{i} \in \mathbb{R}^{d}$. Под асимптотикой оператора $\hat{\rho}_{N}^{t}$, $\operatorname{Tr} \hat{\rho}_{N}^{t}=1$, в [1] подразумевается оператор $\hat{\rho}_{N}^{t, \text { as }}$, аппроксимирующий оператор $\hat{\rho}_{N}^{t}$ :

$$
\left\|\hat{\rho}_{N}^{t, \text { as }}-\hat{\rho}_{N}^{t}\right\|_{1} \rightarrow 0, \quad N \rightarrow \infty, \quad\left\|\hat{\rho}_{N}^{t}\right\|=1,
$$

по следующей норме в пространстве операторов со сходящимся следом в пространстве $L^{2}\left(\mathbb{R}^{d N}\right)$ :

$$
\|\rho\|_{1}=\sup _{O} \frac{|\operatorname{Tr} O \rho|}{\|O\|}
$$

Работа выполнена при финансовой поддержке Российского фонда фундаментальных исследований, грант № 96-01-01544. 
где точная верхняя грань берется по всем ограниченным самосопряженньм операторам $O$, а через $\|O\|$ обозначена операторная норма.

Данная постановка отвечает следующей физической задаче. Имеется система $N$ частищ, находящихся во внешнем поле $U$ и взаимодействуюших между собой с потенциалом $\varepsilon V$. Коэффициент $\varepsilon$, являющийся интенсивностью взаимодействия, обратно пропорционален числу частиц. Оператор $\hat{\rho}_{N}^{t}$ является матрицей плотности системы. Так как при $\operatorname{Tr} \hat{\rho}_{N}=1$ величина $\operatorname{Tr} O_{N} \hat{\rho}_{N}$ является средним значением наблюдаемой $O_{N}$ в состоянии с матрищей плотности $\hat{\rho}_{N}$, то требование $(2)$ означает, что разность средних значений наблюдаемой $O_{N}$ в состояниях $\hat{\rho}_{N}$ и $\hat{\rho}_{N}^{a s}$ стремится при $N \rightarrow \infty$ к 0 , если наблюдаемые $O_{N}$ ограничены равномерно по $N$.

Построенные в работе [1] с помощью теории комплексного ростка [2] в пространстве Фока [3], [4] приближенные решения уравнения (1) имеют ядро $\rho_{N}^{t}\left(x_{1}, \ldots, x_{N} ; y_{1}, \ldots\right.$, $\left.y_{N}\right), x_{i}, y_{i} \in \mathbb{R}^{d}$, симметричное относительно перестановок пар аргументов $\left(x_{i}, y_{i}\right)$, но не обладающее, вообще говоря, свойствами симметрии относительно перестановок аргументов $x_{i}$ и $y_{i}$ по отдельности. В то же время, для матрицы плотности системы $N$ тождественных частиц выполнены определенные свойства симметрии относительно перестановок отдельных аргументов. Ядро матрищы плотности для системы $N$ бозонов симметрично отдельно по $x_{1}, \ldots, x_{N}$ и отдельно по $y_{1}, \ldots, y_{N}$, а для системы фермионов - антисимметрично.

Таким образом, построенные в [1] приближенные решения отвечают системе $N$ различных частиц. В настояшей работе строятся приближенные решения уравнения (1) для систем бозонов и фермионов.

На первьй взгляд, обобщение результатов [1] на случай системы тождественных частищ тривиально: достаточно симметризовать или антисимметризовать ядро матрицы плотности по $x_{1}, \ldots, x_{N}$, так как уравнение Вигнера (1) инвариантно относительно такого преобразования. Однако, как показывается ниже, такой способ построения асимптотик решений уравнения Вигнера приводит, вообще говоря, к ошибочному результату. Это связано с тем, что формулы (2) для нормы $\|\cdot\|_{1}$ неинвариантны относительно симметризации или антисимметризации.

Задача о построении асимптотик решений уравнения (1) для системы тождественных частиц требует качественно нового метода, которьй и развивается в настоящей работе. Соответствующая асимптотика с учетом “обменных эффектов” строится при следующих соотношениях на параметры:

$$
\varepsilon \rightarrow 0, \quad N=O\left(\frac{1}{\varepsilon}\right), \quad \hbar^{d}=O(\varepsilon) .
$$

2. Матрица плотности системы тождественных частиц и вторичное квантование. Асимптотическую формулу для решения задачи Коши для уравнения (1) в случае тождественных частиц удобно строить в представлении вторичного квантования.

ОпредЕЛЕниЕ 1 [1]. Эрмитов оператор $\widehat{\Phi}_{N}^{t}$ в пространстве $L^{2}\left(\mathbb{R}^{d N}\right)$ назьвается полуплотностью, отвечающей матрице плотности $\hat{\rho}_{N}^{t}$, если $\hat{\rho}_{N}^{t}=\left(\widehat{\Phi}_{N}^{t}\right)^{2}$.

В работе [1] доказана 
ЛЕмма 1. Пусть $\Phi_{N}^{t}\left(x_{1}, \ldots, x_{N} ; y_{1}, \ldots, y_{N}\right) u \Phi_{N}^{t, \text { as }}\left(x_{1}, \ldots, x_{N} ; y_{1}, \ldots, y_{N}\right)-я \partial p a$ полуплотностей $\widehat{\Phi}_{N}^{t} u \widehat{\Phi}_{N}^{t, \text { as }}$, отвечающ, их плотностям $\hat{\rho}_{N}^{t} u \hat{\rho}_{N}^{t, \text { as }}$ соответственно. Тогда из свойства

$$
\left\|\Phi_{N}^{t, \text { as }}-\Phi_{N}^{t}\right\|_{L^{2}\left(\mathbb{R}^{2 d N}\right)} \rightarrow 0, \quad N \rightarrow \infty, \quad\left\|\Phi_{N}^{t}\right\|_{L^{2}\left(\mathbb{R}^{2 d N}\right)}^{2}=1,
$$

вытекает свойство (2).

Таким образом, для построения приближенного решения уравнения (1) достаточно построить аппроксимацию для полуплотности по норме $L^{2}\left(\mathbb{R}^{2 d N}\right)$.

Рассмотрим построение асимптотик для системы тождественных частиц. Положим $\sigma= \pm 1$ в зависимости от типа статистики:

$$
\sigma=\left\{\begin{aligned}
1 & \text { в бозе-случае } \\
-1 & \text { в ферми-случае. }
\end{aligned}\right.
$$

Рассмотрим пространство функций $\Phi_{N}\left(x_{1}, \ldots, x_{N} ; y_{1}, \ldots, y_{N}\right)$ из $L^{2}\left(\mathbb{R}^{2 d N}\right)$, удовлетворяющих свойству симметрии относительно перестановок пар $\left(x_{i}, y_{i}\right)$ и соотношению

$$
\begin{aligned}
& \Phi_{N}\left(x_{1}, \ldots, x_{i-1}, x_{j}, x_{i+1}, \ldots, x_{j-1}, x_{i}, x_{j+1}, \ldots, x_{N} ; y_{1}, \ldots, y_{N}\right) \\
& \quad=\sigma \Phi_{N}\left(x_{1}, \ldots, x_{N} ; y_{1}, \ldots, y_{N}\right)
\end{aligned}
$$

как подпространство пространства Фока $\mathscr{F}_{x y}$, состоящего из наборов функций $\Phi=$ $\left\{\Phi_{m n} \in L^{2}\left(\mathbb{R}^{d(m+n)}\right), m, n=0,1,2, \ldots\right\}$, каждая из которых удовлетворяет свойству симметрии:

$$
\begin{aligned}
& \Phi_{m n}\left(x_{1}, \ldots, x_{i-1}, x_{j}, x_{i+1}, \ldots, x_{j-1}, x_{i}, x_{j+1}, \ldots, x_{m} ; y_{1}, \ldots, y_{n}\right) \\
& \quad=\sigma \Phi_{m n}\left(x_{1}, \ldots, x_{m} ; y_{1}, \ldots, y_{n}\right) \\
& \quad \Phi_{m n}\left(x_{1}, \ldots, x_{m} ; y_{1}, \ldots, y_{i-1}, y_{j}, y_{i+1}, \ldots, y_{j-1}, y_{i}, y_{j+1}, \ldots, y_{n}\right) \\
& \quad=\sigma \Phi_{m n}\left(x_{1}, \ldots, x_{m} ; y_{1}, \ldots, y_{n}\right)
\end{aligned}
$$

Введем в пространстве Фока $\mathscr{F} x y$ скалярное произведение

$$
\begin{aligned}
(\Phi, \Psi)= & \sum_{m, n=0}^{\infty} \int d x_{1} \cdots d x_{m} d y_{1} \cdots d y_{n} \Phi_{m n}^{*}\left(x_{1}, \ldots, x_{m} ; y_{1}, \ldots, y_{n}\right) \\
& \times \Psi_{m n}\left(x_{1}, \ldots, x_{m} ; y_{1}, \ldots, y_{n}\right)
\end{aligned}
$$

и операторы рождения и уничтожения $a^{ \pm}(x), b^{ \pm}(y), x, y \in \mathbb{R}^{d}$ :

$$
\begin{gathered}
\left(a^{-}(x) \Phi\right)_{m-1, n}\left(x_{1}, \ldots, x_{m-1} ; y_{1}, \ldots, y_{n}\right)=\sqrt{m} \Phi_{m n}\left(x, x_{1}, \ldots, x_{m-1} ; y_{1}, \ldots, y_{n}\right) \\
\left(a^{+}(x) \Phi\right)_{m n}\left(x_{1}, \ldots, x_{m} ; y_{1}, \ldots, y_{n}\right)=\frac{1}{\sqrt{k}} \sum_{k=1}^{m} \sigma^{k} \delta\left(x-x_{k}\right) \\
\times \Phi_{m-1, n}\left(x_{1}, \ldots, x_{k-1}, x_{k+1}, \ldots, x_{m} ; y_{1}, \ldots, y_{n}\right) \\
\left(b^{-}(y) \Phi\right)_{m, n-1}\left(x_{1}, \ldots, x_{m} ; y_{1}, \ldots, y_{n-1}\right)=\sqrt{n} \sigma^{m+n-1} \\
\times \Phi_{m n}\left(x_{1}, \ldots, x_{m}, y ; y_{1}, \ldots, y_{n-1}\right)
\end{gathered}
$$




$$
\begin{aligned}
\left(b^{+}(y) \Phi\right)_{m n}\left(x_{1}, \ldots, x_{m} ; y_{1}, \ldots, y_{n}\right)=\frac{1}{\sqrt{n}} \sum_{j=1}^{n} \sigma^{m+n-1-j} \\
\quad \times \Phi_{m, n-1}\left(x_{1}, \ldots, x_{m} ; y_{1}, \ldots, y_{j-1}, y_{j+1}, \ldots, y_{n}\right) \delta\left(y-y_{j}\right) .
\end{aligned}
$$

Обозначим через $\Phi^{(0)}$ вакуумный вектор пространства $Ф$ ока, компонента $\Phi_{00}^{(0)}$ которого равна 1, а остальные компоненты равны 0.

Согласно [5] введенные операторы удовлетворяют каноническим перестановочным соотношениям

$$
\begin{gathered}
\left\{a^{ \pm}(x), a^{ \pm}(y)\right\}_{-\sigma}=\left\{a^{ \pm}(x), b^{ \pm}(y)\right\}_{-\sigma}=\left\{a^{ \pm}(x), b^{\mp}(y)\right\}_{-\sigma}=\left\{b^{ \pm}(x), b^{ \pm}(y)\right\}_{-\sigma}=0, \\
\left\{a^{-}(x), a^{+}(y)\right\}_{-\sigma}=\left\{b^{-}(x), b^{+}(y)\right\}_{-\sigma}=\delta(x-y),
\end{gathered}
$$

где через $\{A, B\}_{\sigma}=A B-\sigma B A$ обозначен коммутатор операторов $A, B$ при $\sigma=1$ и антикоммутатор при $\sigma=-1$. Напомним также представление [5] произвольного вектора пространства $\mathscr{F}_{x y}$ через вакуумный вектор и операторы рождения:

$$
\begin{aligned}
\Phi= & \sum_{m n=0}^{\infty} \frac{1}{\sqrt{m ! n !}} \int d x_{1} \cdots d x_{m} d y_{1} \cdots d y_{n} a^{+}\left(x_{1}\right) \cdots a^{+}\left(x_{m}\right) b^{+}\left(y_{n}\right) \cdots b^{+}\left(y_{1}\right) \\
& \times \Phi_{m n}\left(x_{1}, \ldots, x_{m} ; y_{1}, \ldots, y_{n}\right) \Phi^{(0)} .
\end{aligned}
$$

Отождествим полуплотность $\Phi_{N}^{t}$ для системы тождественных частищ с вектором пространства $\Phi$ ока $\Phi^{t}, N$-я компонента которого равна $\Phi_{N}^{t}$, а остальные компоненты равны 0. Частным случаем полученных в [5] результатов является

ЛЕмма 2. Уравнение (1) равносильно следующему уравнению на $\Phi^{t} \in \mathscr{F}_{x y}$ :

$$
i \hbar \frac{d}{d t} \Phi^{t}=L \Phi^{t}
$$

где L - оператор в $\mathscr{F}_{x y}$ вида

$$
\begin{aligned}
L= & \int d x a^{+}(x)\left(-\frac{\hbar^{2}}{2} \frac{\partial^{2}}{\partial x^{2}}+U(x)\right) a^{-}(x)+\frac{\varepsilon}{2} \int d x d y a^{+}(x) a^{+}(y) V(x, y) a^{-}(y) a^{-}(x) \\
& -\int d x b^{+}(x)\left(-\frac{\hbar^{2}}{2} \frac{\partial^{2}}{\partial x^{2}}+U(x)\right) b^{-}(x)-\frac{\varepsilon}{2} \int d x d y b^{+}(x) b^{+}(y) V(x, y) b^{-}(y) b^{-}(x) .
\end{aligned}
$$

Для доказательства леммы 2 достаточно заметить, что уравнение для полуплотности совпадает с уравнением для матрицы плотности (1), и представить через операторы рождения и уничтожения оператор в правой части уравнения (1) согласно [5]. Поскольку скалярное произведение в пространстве $\mathscr{F}_{x y}$ имеет вид (4), задача о построении аппроксимации для полуплотности в смысле (3) равносильна задаче о построении асимптотики решения уравнения (5) по норме пространства $\mathscr{F}_{x y}$ согласно [5]. 
3. Асимптотическая формула для матрицы плотности. Приведем асимптотическую формулу для решения уравнения (5). Пусть $\widehat{\varphi}-$ оператор в пространстве $L^{2}\left(\mathbb{R}^{d}\right)$, имеюший регулярный при $\hbar \rightarrow 0$ вейлевский символ $\varphi(p, q)$. Сопоставим ему оператор $U_{\widehat{\varphi}}$ в пространстве $\mathscr{F}_{x y}$ вида

$$
\begin{aligned}
U_{\widehat{\varphi}}= & \left(\operatorname{det}\left(1-\sigma \hat{\varphi}^{+} \widehat{\varphi}\right)\right)^{\sigma / 2}: \exp W_{\widehat{\varphi}}: \\
W_{\widehat{\varphi}}= & a^{+} \hat{\varphi} b^{+}+a^{+}\left(\left(1-\sigma \hat{\varphi}^{+} \widehat{\varphi}\right)^{1 / 2}-1\right) a^{-} \\
& +b^{+}\left(\left(1-\sigma \widehat{\varphi}^{T} \widehat{\varphi}^{*}\right)^{1 / 2}-1\right) b^{-}+b^{-}\left(1-\sigma \hat{\varphi}^{+} \widehat{\varphi}\right)^{1 / 2} \widehat{\varphi}^{+}\left(1-\sigma \widehat{\varphi} \hat{\varphi}^{+}\right)^{-1 / 2} a^{-},
\end{aligned}
$$

где знак нормального упорядочивания : $\exp W_{\widehat{\varphi}}:$ означает, что после разложения экспоненты $\exp W_{\widehat{\varphi}}$ в ряд операторы рождения в формуле (6) расставлены слева от операторов уничтожения. Через $a^{+} \hat{\varphi} b^{+}$обозначено выражение вида

$$
\int d x a^{+}(x)\left(\widehat{\varphi} b^{+}\right)(x)
$$

аналогичные обозначения приняты и для других слагаемых. Согласно [5] оператор (6) унитарен и однозначно определяется из соотношений

$$
\begin{aligned}
& U_{\widehat{\varphi}}^{-1} a^{-}(x) U_{\widehat{\varphi}}=\left(1-\sigma \widehat{\varphi} \widehat{\varphi}^{+}\right)^{-1 / 2} a^{-}(x)+\widehat{\varphi}\left(1-\sigma \widehat{\varphi}^{+} \widehat{\varphi}\right)^{-1 / 2} b^{+}(x), \\
& U_{\widehat{\varphi}}^{-1} b^{-}(x) U_{\widehat{\varphi}}=\left(1-\sigma \widehat{\varphi}^{T} \widehat{\varphi}^{*}\right)^{-1 / 2} b^{-}(x)+\sigma \widehat{\varphi}^{T}\left(1-\sigma \widehat{\varphi}^{*} \widehat{\varphi}^{T}\right)^{-1 / 2} a^{+}(x), \\
& U_{\widehat{\varphi}}^{-1} a^{+}(x) U_{\widehat{\varphi}}=\left(1-\sigma \widehat{\varphi}^{*} \widehat{\varphi}^{T}\right)^{-1 / 2} a^{+}(x)+\widehat{\varphi}^{*}\left(1-\sigma \widehat{\varphi}^{T} \widehat{\varphi}^{*}\right)^{-1 / 2} b^{-}(x), \\
& U_{\widehat{\varphi}}^{-1} b^{+}(x) U_{\widehat{\varphi}}=\left(1-\sigma \widehat{\varphi}^{+} \hat{\varphi}\right)^{-1 / 2} b^{+}(x)+\sigma \widehat{\varphi}^{+}\left(1-\sigma \widehat{\varphi} \widehat{\varphi}^{+}\right)^{-1 / 2} a^{-}(x)
\end{aligned}
$$

и свойства $\left(\Phi^{(0)}, U_{\widehat{\varphi}} \Phi^{(0)}\right)$. Через $\widehat{\varphi}^{T}$ обозначен оператор, транспонированный $\widehat{\varphi}$, через $\widehat{\varphi}^{*}$ - комплексно сопряженньй к $\widehat{\varphi}$ оператор, через $\widehat{\varphi}^{+}=\left(\widehat{\varphi}^{*}\right)^{T}$ - эрмитово сопряженный к $\widehat{\varphi}$ оператор.

Рассмотрим решение уравнения (5), удовлетворяющее начальному условию вида

$$
\begin{aligned}
\Phi^{\varphi^{0}, M^{0}=} & U_{\widehat{\varphi}^{0}}\left(\operatorname{det}\left(1-\left(M^{0}\right)^{+} M^{0}\right)\right)^{1 / 4} \sum_{n=0}^{n_{0}(\hbar)} \frac{1}{n !}\left(\frac{(2 \pi \hbar)^{d}}{2} \int d x d y a^{+}(x) a^{+}(y)\right. \\
& \left.\times M^{0}\left(-i \hbar \frac{\partial}{\partial x}, x,-i \hbar \frac{\partial}{\partial y}, y\right) b^{+}(y) b^{+}(x)\right)^{n} \Phi^{(0)}
\end{aligned}
$$

где $\widehat{\varphi}^{0}$ - эрмитов оператор в $L^{2}\left(\mathbb{R}^{d}\right),\left(\hat{\varphi}^{0}\right)^{+}=\widehat{\varphi}^{0}$, а $M^{0}\left(p_{1}, q_{1}, p_{2}, q_{2}\right)$ - ядро действующего в $L^{2}\left(\mathbb{R}^{2 d}\right)$ оператора Гильберта-Шмидта $M^{0}$ с нормой меньше 1 . В формуле $(8)$ принято вейлевское [6] упорядочивание операторов

$$
-i \hbar \frac{\partial}{\partial x}, x,-i \hbar \frac{\partial}{\partial y}, y
$$

ЗАмЕчАниЕ 1. Если $M^{0}=0$, то $N$-я компонента вектора (8) пропорциональна симметризованному либо антисимметризованному произведению одночастичных полуплотностей. 
ЗАмечАнИЕ 2. Норма вектора (8) стремится к 1 при $\varepsilon \rightarrow 0, \hbar^{d}=O(\varepsilon)$.

Оказьвается, что решение задачи Коши для уравнения (5) также представляется в виде (8). Пусть $\widehat{\varphi}^{t}$ - решение задачи Коши для уравнения вида

$$
i \hbar \frac{\partial \widehat{\varphi}^{t}}{\partial t}=\left[H\left[\widehat{n}^{t}\right], \widehat{\varphi}^{t}\right]
$$

где через $H[\widehat{n}]$ обозначен оператор в пространстве $L^{2}\left(\mathbb{R}^{d}\right)$ вида

$$
H[\widehat{n}]=-\frac{\hbar^{2}}{2} \frac{\partial^{2}}{\partial x^{2}}+U(x)+\frac{\varepsilon}{(2 \pi \hbar)^{d}} \int V(x, y) n(p, y) d p d y
$$

a $n(p, y)$ - вейлевский символ оператора $n=\widehat{\varphi}^{2}\left(1-\sigma \widehat{\varphi}^{2}\right)^{-1}$.

ЗАмЕчАниЕ. Уравнение (9) равносильно уравнению Хартри-Вигнера

$$
i \hbar \frac{\partial \widehat{n}^{t}}{\partial t}=\left[H\left[\widehat{n}^{t}\right], \widehat{n}^{t}\right]
$$

которое в главном порядке по $\hbar$ совпадает с уравнением Власова. Величина $\widehat{n}$ играет роль плотности частищ. Обозначим через $W$ функцию

$$
W(x)=U(x)+\frac{\varepsilon}{(2 \pi \hbar)^{d}} \int V(x, y) n(p, y) d p d y,
$$

играющую роль самосогласованного потенциала.

Рассмотрим решение задачи Коши для уравнения вида

$$
\begin{aligned}
\left(\frac{\partial}{\partial t}-\left(\frac{\partial W}{\partial q_{1}} \frac{\partial}{\partial p_{1}}-p_{1} \frac{\partial}{\partial q_{1}}+\frac{\partial W}{\partial q_{2}} \frac{\partial}{\partial p_{2}}-p_{2} \frac{\partial}{\partial q_{2}}\right)\right) M^{t}\left(p_{1}, q_{1}, p_{2}, q_{2}\right) \\
=-\int d p_{1}^{\prime} d q_{1}^{\prime} d p_{2}^{\prime} d q_{2}^{\prime} M^{t}\left(p_{1}, q_{1}, p_{1}^{\prime}, q_{1}^{\prime}\right) M^{t}\left(p_{2}, q_{2}, p_{2}^{\prime}, q_{2}^{\prime}\right) \frac{1}{1-\sigma \varphi^{2}\left(p_{1}^{\prime}, q_{1}^{\prime}\right)} \frac{1}{1-\sigma \varphi^{2}\left(p_{2}^{\prime}, q_{2}^{\prime}\right)} \\
\quad \times\left(\frac{\partial V\left(q_{1}^{\prime}, q_{2}^{\prime}\right)}{\partial q_{1}^{\prime}} \frac{\partial}{\partial p_{1}^{\prime}}+\frac{\partial V\left(q_{1}^{\prime}, q_{2}^{\prime}\right)}{\partial q_{2}^{\prime}} \frac{\partial}{\partial p_{2}^{\prime}}\right) \varphi\left(p_{1}^{\prime}, q_{1}^{\prime}\right) \varphi\left(p_{2}^{\prime}, q_{2}^{\prime}\right) \\
+\frac{1}{1-\sigma \varphi^{2}\left(p_{1}, q_{1}\right)} \frac{1}{1-\sigma \varphi^{2}\left(p_{2}, q_{2}\right)}\left(\frac{\partial V\left(q_{1}, q_{2}\right)}{\partial q_{1}} \frac{\partial}{\partial p_{1}}+\frac{\partial V\left(q_{1}, q_{2}\right)}{\partial q_{2}} \frac{\partial}{\partial p_{2}}\right) \varphi\left(p_{1}, q_{1}\right) \varphi\left(p_{2}, q_{2}\right) \\
+\int d p_{1}^{\prime} d q_{1}^{\prime}\left(\frac{1}{1-\sigma \varphi^{2}\left(p_{1}, q_{1}\right)} \frac{\partial}{1-\sigma \varphi^{2}\left(p_{1}^{\prime}, q_{1}^{\prime}\right)}\left(\frac{\partial V\left(q_{1}, q_{1}^{\prime}\right)}{\partial q_{1}} \frac{\partial}{\partial p_{1}}-\frac{\partial V\left(q_{1}, q_{1}^{\prime}\right)}{\partial q_{1}^{\prime}} \frac{\partial}{\partial p_{1}^{\prime}}\right)\right. \\
\left.\quad \times \varphi\left(p_{1}, q_{1}\right) \varphi\left(p_{1}^{\prime}, q_{1}^{\prime}\right)\right) M^{t}\left(p_{1}^{\prime}, q_{1}^{\prime}, p_{2}, q_{2}\right) \\
+\int d p_{2}^{\prime} d q_{2}^{\prime}\left(\frac{1}{1-\sigma \varphi^{2}\left(p_{2}, q_{2}\right)} \frac{\partial V\left(q_{2}, q_{2}^{\prime}\right)}{\partial-\sigma \varphi^{2}\left(p_{2}^{\prime}, q_{2}^{\prime}\right)} \frac{\partial}{\partial p_{2}}-\frac{\partial V\left(q_{2}, q_{2}^{\prime}\right)}{\partial q_{2}^{\prime}} \frac{\partial}{\partial p_{2}^{\prime}}\right) \\
\left.\quad+\varphi\left(p_{2}, q_{2}\right) \varphi\left(p_{2}^{\prime}, q_{2}^{\prime}\right)\right) M^{t}\left(p_{2}^{\prime}, q_{2}^{\prime}, p_{1}, q_{1}\right) .
\end{aligned}
$$

ЗАмЕчАнИЕ. Если в уравнении (11) положить формально $\sigma=0$, то оно перейдет в уравнение типа Риккати, возникающее при построении асимптотик решений многочастичного уравнения Лиувилля [7]. 
Теорема 1. Выполнены соотношения

$$
\left\|\Phi^{t}-\Phi^{\widehat{\varphi}^{t}, M^{t}}\right\| \rightarrow 0, \quad \hbar \rightarrow 0, \varepsilon=\alpha(2 \pi \hbar)^{d}, \quad\left\|\Phi^{t}\right\| \rightarrow 1, \quad \varepsilon \rightarrow 0 .
$$

Таким образом, построена асимптотика решения уравнения (5). Как мы увидим ниже, среднее число частиц порядка $1 / \varepsilon$ :

$$
\frac{1}{\left(\Phi^{t}, \Phi^{t}\right)}\left(\Phi^{t}, \int d x a^{+}(x) a^{-}(x) \Phi^{t}\right)=\frac{1}{(2 \pi \hbar)^{d}} \int d p d q n(p, q)+O(1) .
$$

Оказывается, что свойство (12) справедливо и для $N$-х компонент точной и асимптотической полуплотности, если

$$
N(2 \pi \hbar)^{d}=\int d p d q n(p, q)+O(\varepsilon)
$$

ТЕорема 2. Выполнено свойство

$$
\left\|\frac{\Phi_{N}^{t}}{\left\|\Phi_{N}^{t}\right\|}-\frac{\Phi^{\widehat{\varphi}^{t}, M^{t}}}{\left\|\Phi_{N}^{t}\right\|}\right\| \rightarrow 0, \quad \hbar \rightarrow 0, \quad \varepsilon=\alpha(2 \pi \hbar)^{d} .
$$

Из теоремы 2 вытекает

СлЕДСтвиЕ. Пусть $\rho_{N}^{t}-$ решение уравнения (1), удовлетворяющее начальному условию

$$
\rho_{N}^{0}=\frac{\left(\Phi_{N}^{0}\right)^{2}}{\left\|\Phi_{N}^{0}\right\|^{2}}
$$

Тогда для

$$
\rho_{N, \mathrm{as}}^{t}=\frac{\left(\Phi_{N}^{\varphi^{t}, M^{t}}\right)^{2}}{\left\|\Phi_{N}^{t}\right\|^{2}}
$$

справедливо свойство (2).

Доказательства теорем 1 и 2 приводятся ниже.

Уравнение (11) можно привести к более простому виду. Рассмотрим оператор $B^{t}$, связанньй с $M^{t}$ соотношением

$$
M^{t}=\left(1+\varphi^{t} B^{t} \varphi^{t}\right)^{-1}\left(1-\varphi^{t} B^{t} \varphi^{t}\right),
$$

через $\varphi^{t} B^{t} \varphi^{t}$ обозначен оператор в $L^{2}\left(\mathbb{R}^{2 d}\right)$ с ядром $\varphi^{t}\left(p_{1}, q_{1}\right) B^{t}\left(p_{1}, q_{1}, p_{2}, q_{2}\right) \varphi^{t}\left(p_{2}, q_{2}\right)$. Ядро оператора $B^{t}$ удовлетворяет уравнению

$$
\begin{aligned}
\frac{\partial}{\partial t} B^{t}\left(p_{1}, q_{1}, p_{2}, q_{2}\right)= & \left(\frac{\partial W}{\partial q_{1}} \frac{\partial}{\partial p_{1}}-p_{1} \frac{\partial}{\partial q_{1}}+\frac{\partial W}{\partial q_{2}} \frac{\partial}{\partial p_{2}}-p_{2} \frac{\partial}{\partial q_{2}}\right) B^{t}\left(p_{1}, q_{1}, p_{2}, q_{2}\right) \\
& -\int d p_{1}^{\prime} d q_{1}^{\prime} \frac{\partial n\left(p_{1}^{\prime}, q_{1}^{\prime}\right)}{\partial p_{1}^{\prime}} \frac{\partial V\left(q_{1}, q_{1}^{\prime}\right)}{\partial q_{1}^{\prime}} B^{t}\left(p_{1}^{\prime}, q_{1}^{\prime}, p_{2}, q_{2}\right) \\
& -\int d p_{2}^{\prime} d q_{2}^{\prime} \frac{\partial n\left(p_{2}^{\prime}, q_{2}^{\prime}\right)}{\partial p_{2}^{\prime}} \frac{\partial V\left(q_{2}, q_{2}^{\prime}\right)}{\partial q_{2}^{\prime}} B^{t}\left(p_{1}, q_{1}, p_{2}^{\prime}, q_{2}^{\prime}\right) .
\end{aligned}
$$


ЗАмечАниЕ. Уравнения $(10),(13)$ на $n^{t}(p, q)$ и $B^{t}\left(p_{1}, q_{1}, p_{2}, q_{2}\right)$ не зависят от типа статистики и совпадают с аналогичньми уравнениями для классической статистики [7]. Поэтому может сложиться впечатление, что тождественность частиц не сказьвается при построении асимптотики. Однако, условие квадратичной интегрируемости функции

$$
\frac{n\left(p_{1}, q_{1}\right)}{1+\sigma n\left(p_{1}, q_{1}\right)} B^{t}\left(p_{1}, q_{1}, p_{2}, q_{2}\right) \frac{n\left(p_{2}, q_{2}\right)}{1+\sigma n\left(p_{2}, q_{2}\right)}-\delta\left(p_{1}-p_{2}\right) \delta\left(q_{1}-q_{2}\right),
$$

которое накладывается на $B^{t}$ в силу условия $\operatorname{Tr} M^{+} M<\infty$, зависит от типа статистики. Таким образом, одному классу решений уравнения (12) отвечают приближенные решения бозонного уравнения Вигнера, другому классу решений уравнения (12) - асимптотики в фермионном случае.

4. Пример стационарного асимптотического решения уравнения Вигнера. Приведем пример решений уравнений (10) и (11). Рассмотрим не зависящий от $t$ оператор $\widehat{\varphi}$ вида

$$
\widehat{\varphi}=\exp \left(-\frac{1}{2 \theta}\left(-\frac{\hbar^{2}}{2} \frac{\partial^{2}}{\partial x^{2}}+W(x)-\mu\right)\right)
$$

где $\theta$ и $\mu$-вещественные параметры, а $W$ - гладкая функция. Оператор (14) удовлетворяет уравнению (9), если

$$
W(x)=U(x)+\alpha \int V(x, y) n(p, y) d p d y
$$

где $n(p, y)$ - вейлевский символ оператора

$$
\widehat{n}=\left(\exp \left(\frac{1}{\theta}\left(-\frac{\hbar^{2}}{2} \frac{\partial^{2}}{\partial x^{2}}+W(x)-\mu\right)\right)-\sigma\right)^{-1}
$$

который в главном порядке по $\hbar$ имеет вид

$$
n(p, y)=\left(\exp \left(\frac{1}{\theta}\left(-\frac{p^{2}}{2}+W(x)-\mu\right)\right)-\sigma\right)^{-1}+O(\hbar) .
$$

Рассмотрим построение стационарного решения уравнения (11) в главном порядке по $\hbar$. Обозначим $f=\varphi /\left(1-\sigma \varphi^{2}\right)$. Функция

$$
M\left(p_{1}, q_{1}, p_{2}, q_{2}\right)=f\left(p_{1}, q_{1}\right) f\left(p_{2}, q_{2}\right) \widetilde{M}\left(q_{1}, q_{2}\right)
$$

удовлетворяет уравнению (11), если

$$
\frac{\partial}{\partial q_{1}}\left(\widetilde{M}\left(q_{1}, q_{2}\right)-\frac{\alpha}{2 \theta} V\left(q_{1}, q_{2}\right)-\frac{\alpha}{2 \theta} \int d p_{1}^{\prime} d q_{1}^{\prime} V\left(q_{1}, q_{1}^{\prime}\right) f^{2}\left(p_{1}^{\prime}, q_{1}^{\prime}\right) \widetilde{M}\left(q_{1}^{\prime}, q_{2}\right)\right)=0 .
$$

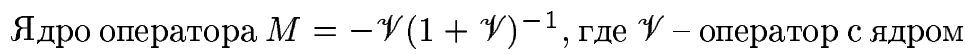

$$
\mathscr{V}\left(p_{1}, q_{1}, p_{2}, q_{2}\right)=\frac{\alpha}{2 \theta} f\left(p_{1}, q_{1}\right) V\left(q_{1}, q_{2}\right) f\left(p_{2}, q_{2}\right),
$$

удовлетворяет соотношению (15).

Из теорем 1 и 2 вытекает 
СлЕДСТВИЕ. Для решения $\Phi^{t}$ уравнения (5), удовлетворяющего начальному условию

$$
\Phi^{0}=\Phi^{\widehat{\varphi}^{0}, M^{0}}
$$

выполнено соотношение

$$
\left\|\Phi^{t}-\Phi^{0}\right\| \rightarrow 0, \quad \hbar \rightarrow 0, \quad \varepsilon=\alpha(2 \pi \hbar)^{d}, \quad t=\text { const } .
$$

Таким образом, мы построили стационарные асимптотические решения уравнения для матрицы плотности. В [8] приведены соображения, показывающие, что формула (16) аппроксимирует большое каноническое распределение Гиббса.

5. Оценка нормы асимптотической формулы. Для доказательства асимптотической формулы для решения задачи Коши рассмотрим оценку нормы этой формулы сверху и снизу. Поскольку оператор $U_{\varphi}$ унитарен, достаточно рассмотреть вектор пространства Фока вида

$$
Y_{M}=\sum_{n=0}^{n_{0}(\hbar)} Y_{M}^{(n)}
$$

где

$$
Y_{M}^{(n)}=\frac{(2 \pi \hbar)^{d n}}{2^{n} n !}\left(\int d x d y a^{+}(x) a^{+}(y) M\left(-i \hbar \frac{\partial}{\partial x}, x,-i \hbar \frac{\partial}{\partial y}, y\right) b^{+}(y) b^{+}(x)\right)^{n} \Phi^{(0)}
$$

Поскольку квадрат нормы нулевой компоненты вектора (17) равен 1, справедлива оценка $\left\|Y_{M}\right\| \geqslant 1$. Для оценки нормы вектора (17) сверху рассмотрим $n$-ю компоненту этого вектора (18).

ЛЕмма 3. Для некоторых не зависящих от $n$ положительных констант $a, b, c$ выполнено соотношение

$$
\left\|Y_{M}^{(n)}\right\|^{2} \leqslant b e^{-c n} \sum_{k=0}^{n} k ! a^{k} \hbar^{d k / 2}
$$

Разобьем доказательство этой формулы на ряд последовательных утверждений.

Рассмотрим вспомогательноепространство Фока $\widetilde{\mathscr{F}}$, состоящее из наборов комплексных функций $\left(\varphi_{0}, \varphi_{1}, \ldots, \varphi_{n}, \ldots\right)$, каждая из которых $\varphi_{n}\left(x_{1}, y_{1}, \ldots, x_{n}, y_{n}\right), x_{1}, \ldots, x_{n}$, $y_{1}, \ldots, y_{n} \in \mathbb{R}^{d}$, симметрична относительно перестановок пар аргументов $\left(x_{i}, y_{i}\right)$. В пространстве $\widetilde{\mathscr{F}}$ введем норму

$$
\|\varphi\|^{2}=\sum_{n=0}^{\infty}\left\|\varphi_{n}\right\|_{L^{2}\left(\mathbb{R}^{2 d n}\right)}^{2}
$$

и операторы рождения и уничтожения согласно [5]:

$$
\begin{aligned}
&\left(A^{-}(x, y) \varphi\right)_{n-1}\left(x_{1}, y_{1}, \ldots, x_{n-1}, y_{n-1}\right)=\sqrt{n} \varphi_{n}\left(x, y, x_{1}, y_{1}, \ldots, x_{n-1}, y_{n-1}\right) \\
&\left(A^{+}(x, y) \varphi\right)_{n}\left(x_{1}, y_{1}, \ldots, x_{n}, y_{n}\right)=\frac{1}{\sqrt{n}} \sum_{k=1}^{n} \delta\left(x-x_{k}\right) \delta\left(y-y_{k}\right) \\
& \times \varphi_{n-1}\left(x_{1}, y_{1}, \ldots, x_{k-1}, y_{k-1}, x_{k+1}, y_{k+1}, \ldots, x_{n}, y_{n}\right) .
\end{aligned}
$$


Через $\widetilde{\varphi}^{(0)}$ мы обозначаем вакуумньй вектор пространства $\widetilde{\mathscr{F}}$, нулевая компонента которого равна 1 , а остальные - 0 .

Рассмотрим оператор симметризации (или антисимметризации) $W: \widetilde{\mathscr{F}} \rightarrow \mathscr{F}_{x y}$, переводящий вектор пространства $\widetilde{\mathscr{F}}$ с компонентами $\left(\varphi_{0}, \varphi_{1}, \ldots, \varphi_{n}, \ldots\right)$ в вектор пространства $Ф_{\text {ока }} \mathscr{F}_{x y}$ с компонентами вида

$$
\Phi_{n n}\left(x_{1}, \ldots, x_{n}, y_{1}, \ldots, y_{n}\right)=\frac{1}{\sqrt{n !}} \sum_{\alpha} \sigma^{P(\alpha)} \varphi_{n}\left(x_{1}, y_{\alpha_{1}}, \ldots, x_{n}, y_{\alpha_{n}}\right)
$$

и остальными компонентами, равными 0. В формуле (20) производится суммирование по всем перестановкам $\alpha$ чисел $1, \ldots, n$, через $P(\alpha)$ обозначена четность перестановки.

Сопоставим [5] каждому полиномиальному функционалу функций $\alpha^{*}$ и $\alpha$

$$
\begin{aligned}
F\left(\alpha^{*}, \alpha\right)= & \sum_{m n} \int d \xi_{1} \cdots d \xi_{m} d \eta_{1} \cdots d \eta_{n} \alpha^{*}\left(\xi_{1}\right) \cdots \alpha^{*}\left(\xi_{m}\right) \\
& \times \alpha\left(\eta_{1}\right) \cdots \alpha\left(\eta_{n}\right) F^{m n}\left(\xi_{1}, \ldots, \xi_{m}, \eta_{1}, \ldots, \eta_{n}\right),
\end{aligned}
$$

где $\xi_{i}=\left(x_{i}, y_{i}\right) \in \mathbb{R}^{2 d}, \eta_{i}=\left(x_{i}^{\prime}, y_{i}^{\prime}\right) \in \mathbb{R}^{2 d}$, оператор в пространстве $\mathscr{F}_{x y}$ вида

$$
\begin{aligned}
: F\left(A^{+}, A^{-}\right):= & \sum_{m n} \int d \xi_{1} \cdots d \xi_{m} d \eta_{1} \cdots d \eta_{n} A^{+}\left(\xi_{1}\right) \cdots A^{+}\left(\xi_{m}\right) \\
& \times A^{-}\left(\eta_{1}\right) \cdots A^{-}\left(\eta_{n}\right) F^{m n}\left(\xi_{1}, \ldots, \xi_{m}, \eta_{1}, \ldots, \eta_{n}\right) .
\end{aligned}
$$

Функционал (21) называется нормальным символомоператора (22) (см. [5]). Сопоставим функции $\alpha(x, y)$ оператор $\widehat{\alpha}$ в пространстве $L^{2}\left(\mathbb{R}^{2 d}\right)$ с ядром $\alpha(x, y)$.

Пусть $n$-я компонента вектора $\varphi \in \widetilde{\mathscr{F}}$ вещественна и отлична от 0 , а остальные компоненты равны 0 .

ЛЕмма 4. Квадрат нормы вектора $W \varphi \in \mathscr{F}_{x y}$

$$
\|W \varphi\|^{2}=\sum_{k=0}^{n} \sum_{2 n_{2}+3 n_{3}+\cdots=k} \frac{\sigma^{n_{2}+n_{4}+n_{6}+\cdots}}{2^{n_{2}} n_{2} ! 3^{n_{3}} n_{3} ! \cdots}\left(\varphi, \widehat{W}_{n_{2} \ldots n_{k}} \varphi\right)
$$

вырахсается через матричные әлементы $\left(\varphi, \widehat{W}_{n_{2} \ldots n_{k}} \varphi\right)$ операторов $\widehat{W}_{n_{2} \ldots n_{k}} c$ нормальными символами

$$
W_{n_{2} \ldots n_{k}}\left(\alpha^{*}, \alpha\right)=\prod_{l=2}^{k}\left(\operatorname{Tr}\left(\alpha^{*} \alpha\right)^{l}\right)^{n_{l}}
$$

ДокАЗАТЕльСТво. В силу формулы (20) квадрат нормы представляется в виде следующей суммы по перестановкам $\alpha, \beta$ :

$$
\begin{aligned}
\|W \varphi\|^{2}= & \frac{1}{n !} \sum_{\alpha \beta} \sigma^{P(\alpha)+P(\beta)} \int d x_{1} \cdots d x_{n} d y_{1} \cdots d y_{n} \varphi_{n}\left(x_{1}, y_{\alpha_{1}}, \ldots, x_{n}, y_{\alpha_{n}}\right) \\
& \times \varphi_{n}\left(x_{1}, y_{\beta_{1}}, \ldots, x_{n}, y_{\beta_{n}}\right) .
\end{aligned}
$$


Рассматривая замену переменных $z_{1}=y_{\alpha_{1}}, \ldots, z_{n}=y_{\alpha_{n}}$ и обозначая перестановку $\beta \alpha^{-1}$ через $\gamma, \beta_{i}=\gamma_{\alpha_{i}}$, получаем

$$
\begin{aligned}
\|W \varphi\|^{2}= & \frac{1}{n !} \sum_{\alpha \gamma} \sigma^{P(\gamma)} \int d x_{1} \cdots d x_{n} d z_{1} \cdots d z_{n} \varphi_{n}\left(x_{1}, z_{1}, \ldots, x_{n}, z_{n}\right) \\
& \times \varphi_{n}\left(x_{1}, z_{\gamma_{1}}, \ldots, x_{n}, z_{\gamma_{n}}\right) .
\end{aligned}
$$

Выражение, стоящее под знаком суммы, не зависит от $\alpha$, поэтому суммирование

$$
\frac{1}{n !} \sum_{\alpha \gamma}
$$

можно заменить на $\sum_{\gamma}$. В силу свойства [5]

$$
\varphi_{n}\left(x_{1}, z_{1}, \ldots, x_{n}, z_{n}\right) \tilde{\varphi}^{(0)}=\frac{1}{\sqrt{n !}} A^{-}\left(x_{1}, z_{1}\right) \cdots A^{-}\left(x_{n}, z_{n}\right) \varphi
$$

получаем

$$
\begin{aligned}
\|W \varphi\|^{2}= & \frac{1}{n !} \sum_{\gamma} \sigma^{P(\gamma)} \int d x_{1} \cdots d x_{n} d z_{1} \cdots d z_{n} \\
& \times\left(\varphi, A^{+}\left(x_{1}, z_{1}\right) \cdots A^{+}\left(x_{n}, z_{n}\right) A^{-}\left(x_{n}, z_{\gamma_{n}}\right) \cdots A^{-}\left(x_{1}, z_{\gamma_{1}}\right) \varphi\right) .
\end{aligned}
$$

Известно (см., например, [9]), что любая перестановка распадается на произведение независимых циклов. Пусть перестановка $\gamma$ содержит $n_{1}$ цикл длины $1, n_{2}$ цикла длины 2 , и т.д., причем $n_{1}+2 n_{2}+3 n_{3}+\cdots=n$. Перестановки с заданными $\left(n_{1}, n_{2}, \ldots\right)$ дают одинаковый вкладв сумму (24). Найдем количество таких перестановок: $n$ чисел можно разбить на $n_{1}$ групп из одного числа, $n_{2}$ групп из двух чисел и т.д.

$$
\frac{n !}{1^{n_{1}}(2 !)^{n_{2}}(3 !)^{n_{3}} \cdots} \frac{1}{n_{1} ! n_{2} ! n_{3} ! \cdots}
$$

способами. Существует $\left(n_{k}-1\right)$ ! способов циклической перестановки $n_{k}$ чисел. Таким образом, число перестановок с заданными $\left(n_{1}, n_{2}, \ldots\right)$ равно

$$
\frac{n !}{n_{1} ! 2^{n_{2}} n_{2} ! 3^{n_{3}} n_{3} ! \cdots}
$$

Не ограничивая общности, можно считать, что $n_{1}$ индексов, остающиеся инвариантными, равны $n-n_{1}+1, \ldots, n$. Обозначая $k=n-n_{1}$, получаем

$$
\begin{aligned}
\|W \varphi\|^{2}= & \sum_{k=0}^{n} \sum_{2 n_{2}+3 n_{3} \cdots=k} \frac{1}{2^{n_{2} n_{2} ! 3^{n_{3}} n_{3} ! \cdots}} \sigma^{P(\gamma)} \int d x_{1} \cdots d x_{k} d z_{1} \cdots d z_{k} \\
& \times\left(\varphi, A^{+}\left(x_{1}, z_{1}\right) \cdots A^{+}\left(x_{k}, z_{k}\right) I_{n-k} \frac{1}{(n-k) !} A^{-}\left(x_{k}, z_{\gamma_{k}}\right) \cdots A^{-}\left(x_{1}, z_{\gamma_{1}}\right) \varphi\right),
\end{aligned}
$$


где через $I_{n-k}$ обозначен оператор

$$
\begin{aligned}
I_{n-k}= & \int d x_{k+1} \cdots d x_{n} d z_{k+1} \cdots d z_{n} A^{+}\left(x_{k+1}, z_{k+1}\right) \cdots A^{+}\left(x_{n}, z_{n}\right) \\
& \times A^{-}\left(x_{n}, z_{n}\right) \cdots A^{-}\left(x_{k+1}, z_{k+1}\right) .
\end{aligned}
$$

Поскольку при действии на $(n-k)$-ю компоненту вектора пространства Фока оператор $I_{n-k}$ умножает его на $(n-k)$ !, а четность перестановки $\gamma$ равна $n_{2}+n_{4}+\cdots$, формула (25) приводится к виду

$$
\begin{aligned}
\|W \varphi\|^{2}= & \sum_{k=0}^{n} \sum_{2 n_{2}+3 n_{3} \cdots=k} \frac{1}{2^{n_{2}} n_{2} ! 3^{n_{3}} n_{3} ! \cdots} \sigma^{n_{2}+n_{4}+\cdots} \int d x_{1} \cdots d x_{k} d z_{1} \cdots d z_{k} \\
& \times\left(\varphi, A^{+}\left(x_{1}, z_{1}\right) \cdots A^{+}\left(x_{k}, z_{k}\right) A^{-}\left(x_{k}, z_{\gamma_{k}}\right) \cdots A^{-}\left(x_{1}, z_{\gamma_{1}}\right) \varphi\right) .
\end{aligned}
$$

Формула (26) совпадает с (23). Лемма 4 доказана.

Из леммы 4 вытекает формула для квадрата нормы вектора (18). Обозначим через $\xi$ совокупность аргументов $(x, y)$, через $\widetilde{M}$ - оператор в пространстве $L^{2}\left(\mathbb{R}^{2 d}\right)$ с ядром вида

$$
\begin{aligned}
\widetilde{M}\left(x_{1}, y_{1}, x_{2}, y_{2}\right)= & \frac{1}{(2 \pi \hbar)^{d}} \int d p_{1} d p_{2} M\left(p_{1}, \frac{x_{1}+y_{1}}{2}, p_{2}, \frac{x_{2}+y_{2}}{2}\right) \\
& \times \exp \left(\frac{i}{\hbar}\left(p_{1}\left(x_{1}-y_{1}\right)+p_{2}\left(x_{2}-y_{2}\right)\right)\right)
\end{aligned}
$$

удовлетворяющий свойству $\|\widetilde{M}\|<1$. Введем операторы

$$
B_{M}^{ \pm}(\xi)=\left(1-\widetilde{M}^{+} \widetilde{M}\right)^{-1 / 2} A^{ \pm}(\xi)+\widetilde{M}\left(1-\widetilde{M}^{+} \widetilde{M}\right)^{-1 / 2} A^{\mp}(\xi) .
$$

Лемма 5. Для любого $c>0$ такого, что $e^{c / 2}\|\widetilde{M}\|<1$, справедливо соотношенuе

$$
\begin{aligned}
& \left\|Y_{M}^{(n)}\right\|^{2}=\frac{e^{-c n}}{2 \pi \sqrt{\operatorname{det}\left(1-e^{c} \widetilde{M}^{2}\right)}} \sum_{k=0}^{n} \sum_{2 n_{2}+3 n_{3}+\cdots=k} \frac{\sigma^{n_{2}+n_{4}+\cdots}}{2^{n_{2}} n_{2} ! 3^{n_{3}} n_{3} ! \cdots} \int_{0}^{2 \pi} d \zeta e^{-2 i \zeta n} \\
& \times \int d x_{1} \cdots d x_{k} d z_{1} \cdots d z_{k}\left(\widetilde{\varphi}^{(0)}, B_{M e^{c / 2+i \zeta}}^{+}\left(x_{1}, z_{1}\right) \cdots B_{M e^{c / 2+i \zeta}}^{+}\left(x_{k}, z_{k}\right)\right. \\
& \left.\times B_{M e^{c / 2+i \zeta}}^{-}\left(x_{k}, z_{\gamma_{k}}\right) \cdots B_{M e^{c / 2+i \zeta}}^{-}\left(x_{1}, z_{\gamma_{1}}\right) \tilde{\varphi}^{(0)}\right) \text {. }
\end{aligned}
$$

ДокАЗАТЕльство. Так как $n$-я компонента вектора $Y_{M}$ может быть представлена в виде $Y_{M}^{(n)}=W P_{n} \varphi_{M}$, где $P_{n}$ - оператор выделения $n$-й компоненты из вектора

$$
\varphi_{M}=\exp \left(\frac{1}{2} \int d \xi d \eta \widetilde{M}(\xi, \eta) A^{+}(\xi) A^{+}(\eta)\right) \widetilde{\varphi}^{(0)}
$$

то в соответствии с леммой 4 квадрат нормы $\left\|Y_{M}^{(n)}\right\|^{2}$ имеет вид $(26)$ при $\varphi=P_{n} \varphi_{M}$. Матричный элемент $\left(P_{n} \varphi_{M}, \widehat{W}_{n_{2} \ldots n_{k}} P_{n} \varphi_{M}\right)$ может быть представлен в виде

$$
\int_{0}^{2 \pi} \frac{d \zeta}{2 \pi} e^{-c n} e^{-2 i \zeta n}\left(P_{n} \varphi_{M e^{c / 2+i \zeta}}, \widehat{W}_{n_{2} \ldots n_{k}} P_{n} \varphi_{M e^{c / 2+i \zeta}}\right)
$$


Согласно [5] вектор $\varphi_{M}$ может быть выражен через оператор унитарного преобразования $U_{M}$, связьвающего операторы $A^{ \pm}(\xi)$ и $B^{ \pm}(\xi)$,

$$
U_{M}^{+} A^{ \pm}(\xi) U_{M}=B^{ \pm}(\xi)
$$

и вакуумный вектор $\widetilde{\varphi}^{(0)}$,

$$
\varphi_{M}=\left(\operatorname{det}\left(1-M^{+} M\right)\right)^{-1 / 4} U_{M} \widetilde{\varphi}^{(0)} .
$$

Подставляя приведенные формулы в формулу (26), получаем утверждение леммы 5.

Для оценки матричного элемента, входящего в формулу (28), введем некоторые вспомогательные понятия.

Назовем вектор пространства Фока $\widetilde{\mathscr{F}}$ с неотрицательными вещественными компонентами неотрицательным. Будем говорить, что оператор $B: \widetilde{\mathscr{F}} \rightarrow \widetilde{\mathscr{F}}$ неотрииателен, $B \geqslant 0$, если он переводит любой неотрицательный вектор в неотрицательный. Введем на множестве операторов, действуюших в $\widetilde{\mathscr{F}}$, отношение частичного порядка: будем говорить, что $B_{1} \geqslant B_{2}, B_{1}, B_{2}: \widetilde{\mathscr{F}} \rightarrow \widetilde{\mathscr{F}}$, если $B_{1}-B_{2} \geqslant 0$. Назовем модулем оператора $B$,

$$
(B \varphi)_{m}\left(\xi_{1}, \ldots, \xi_{m}\right)=\sum_{n} \int d \eta_{1} \cdots d \eta_{n} B_{m n}\left(\zeta_{1}, \ldots, \zeta_{m}, \eta_{1}, \ldots, \eta_{n}\right) \varphi_{n}\left(\eta_{1}, \ldots, \eta_{n}\right),
$$

оператор $|B|$ вида

$$
(|B| \varphi)_{m}\left(\xi_{1}, \ldots, \xi_{m}\right)=\sum_{n} \int d \eta_{1} \cdots d \eta_{n}\left|B_{m n}\left(\zeta_{1}, \ldots, \zeta_{m}, \eta_{1}, \ldots, \eta_{n}\right)\right| \varphi_{n}\left(\eta_{1}, \ldots, \eta_{n}\right)
$$

Непосредственно из введенных определений вытекает

Лемма 6. Выполнены соотношения:

1) $\left|B_{1} B_{2}\right| \leqslant\left|B_{1}\right|\left|B_{2}\right| ;\left|\left(\widetilde{\varphi}^{(0)}, B \widetilde{\varphi}^{(0)}\right)\right| \leqslant\left(\widetilde{\varphi}^{(0)},|B| \widetilde{\varphi}^{(0)}\right)$;

2) $n p u|F|<G$

$$
\left|\int d \eta F(\xi, \eta) A^{ \pm}(\eta)\right| \leqslant \int d \eta G(\xi, \eta) A^{ \pm}(\eta)
$$

Получим оценку сверху для ядер операторов

$$
\left(1-e^{c} \widetilde{M}^{2}\right)^{-1 / 2}-1, \quad e^{c / 2} \widetilde{M}\left(1-e^{c} \widetilde{M}^{2}\right)^{-1 / 2} e^{i \zeta}
$$

Пусть

$$
\phi(x, y)=\frac{1}{(2 \pi \hbar)^{d / 2}} \chi\left(\frac{x-y}{\hbar}, \frac{x+y}{2}\right)>0,
$$

причем гладкая функция $1 / \chi(\rho, z)$ растет при $\rho, z \rightarrow \infty$ не быстрее полинома.

ЛЕмма 7. Для некоторой не зависящей от ћ константы ядра операторов (29) не превосходят $a_{1} \phi(\xi) \phi(\eta), \xi=\left(x_{1}, y_{1}\right) \in \mathbb{R}^{2 d}, \eta=\left(x_{2}, y_{2}\right) \in \mathbb{R}^{2 d}$. 
ДокАЗАТЕльСтво. По теореме вложения Соболева (см, например, [10]) достаточно проверить, что ядра операторов

$$
\frac{1}{\phi}\left(\left(1-e^{c} \widetilde{M}^{2}\right)^{-1 / 2}-1\right) \frac{1}{\phi}, \quad \frac{1}{\phi} \widetilde{M} e^{c / 2} e^{i \zeta}\left(1-e^{c} \widetilde{M}^{2}\right)^{-1 / 2} \frac{1}{\phi}
$$

принадлежат при любом $k$ пространству $W_{2}^{k}\left(\mathbb{R}^{4 d}\right)$. Для этого проверим, что операторы

$$
\begin{gathered}
(-\Delta+1)^{k / 2} \frac{1}{\phi}\left(\left(1-e^{c} \widetilde{M}^{2}\right)^{-1 / 2}-1\right) \frac{1}{\phi}(-\Delta+1)^{k / 2} \\
(-\Delta+1)^{k / 2} \frac{1}{\phi} \widetilde{M}\left(1-e^{c} \widetilde{M}^{2}\right)^{-1 / 2} \frac{1}{\phi}(-\Delta+1)^{k / 2}
\end{gathered}
$$

где

$$
\Delta=\frac{\partial^{2}}{\partial \rho^{2}}+\frac{\partial^{2}}{\partial z^{2}}, \quad \rho=\frac{x-y}{\hbar}, \quad z=\frac{x+y}{2},
$$

являются операторами Гильберта-Шмидта. Данное утверждение, в свою очередь, доказывается разложением выражения $\left(1-e^{c} \widetilde{M}\right)^{-1 / 2}$ в сходящийся по операторной норме степенной ряд и использованием соотношений типа

$$
\|A B\|_{2} \leqslant\|A\|\|B\|_{2}, \quad\|B\|_{2}=\sqrt{\operatorname{Tr} B^{+B}} .
$$

Лемма 7 доказана.

Используя леммы 6 и 7 , получаем следствие из леммы 5 .

СледСтвиЕ. Справедлива оченка

$$
\begin{aligned}
\left\|Y_{M}^{(n)}\right\|^{2} \leqslant & \frac{e^{-c n}}{\sqrt{\operatorname{det}\left(1-e^{c} \widetilde{M}^{2}\right)}} \sum_{k=0}^{n} \sum_{2 n_{2}+3 n_{3}+\cdots=k} \frac{1}{2^{n_{2} n_{2} ! 3^{n_{3}} n_{3} ! \cdots} \int d x_{1} \cdots d x_{k} d z_{1} \cdots d z_{k}} \\
& \times\left(\widetilde{\varphi}^{(0)}, C^{+}\left(x_{1}, z_{1}\right) \cdots C^{+}\left(x_{k}, z_{k}\right) C^{-}\left(x_{k}, z_{\gamma_{k}}\right) \cdots C^{-}\left(x_{1}, z_{\gamma_{1}}\right) \widetilde{\varphi}^{(0)}\right),
\end{aligned}
$$

əде

$$
C^{ \pm}(\xi)=A^{+}(\xi)+a_{1}\left(A^{+}[\phi]+A^{-}[\phi]\right) \phi(\xi), \quad A^{ \pm}[\phi]=\int d \xi A^{ \pm}(\xi) \phi(\xi)
$$

ДокАЗАТЕЛЬСтво ЛЕммы 3. Так как операторы

$$
\widetilde{A}^{ \pm}(\xi)=A^{ \pm}(\xi)-A^{ \pm}[\phi] \phi(\xi)\left(\int d \xi|\phi(\xi)|^{2}\right)^{-1 / 2}
$$

коммутируют с операторами $A^{ \pm}(\xi)$, а операторы уничтожения переводят вакуумный вектор в 0 , операторы $C^{ \pm}(\xi)$ в формуле (31) могут быть заменены на операторы вида

$$
\widetilde{C}^{ \pm}(\xi)=\phi(\xi)\left(A^{ \pm}[\phi]\left(a_{1}+\left(\int d \xi|\phi(\xi)|^{2}\right)^{-1}\right)+A^{\mp}[\phi] a_{1}\right) .
$$


Далее, если вектор $\psi \in \widetilde{\mathscr{F}}$ содержит только $s$ компонент, то $\left\|A^{ \pm}[\phi] \psi\right\| \leqslant \sqrt{s}\|\phi\|\|\psi\|$. Используя данное соотношение, приходим к оценке

$$
\begin{aligned}
& \left\|Y_{M}^{(n)}\right\|^{2} \leqslant \frac{e^{-c n}}{\sqrt{\operatorname{det}\left(1-e^{c} \widetilde{M}^{2}\right)}} \sum_{k=0}^{n} \sum_{2 n_{2}+3 n_{3}+\cdots=k} \frac{1}{2^{n_{2}} n_{2} ! 3^{n_{3}} n_{3} ! \cdots} \int d x_{1} \cdots d x_{k} d z_{1} \cdots d z_{k} \\
& \quad \times\left(\widetilde{\varphi}^{(0)}, \phi\left(x_{1}, z_{1}\right) \cdots \phi\left(x_{k}, z_{k}\right) \phi\left(x_{k}, z_{\gamma_{k}}\right) \cdots \phi\left(x_{1}, z_{\gamma_{1}}\right) \widetilde{\varphi}^{(0)}\right)\left(2\left(a_{1}+\|\phi\|^{-2}\right)\right)^{2 k} k !\|\phi\|^{2 k} .
\end{aligned}
$$

Отсюда для некоторых $a, b, c$ получаем

$$
\left\|Y_{M}^{(n)}\right\|^{2} \leqslant b e^{-c n} \sum_{k=0}^{n} k ! a_{2}^{k} \sum_{2 n_{2}+3 n_{3}+\cdots=k} \frac{1}{2^{n_{2} n_{2} ! 3^{n_{3}} n_{3} ! \cdots}}\left(\operatorname{Tr} \widehat{\phi}^{4}\right)^{n_{2}}\left(\operatorname{Tr} \widehat{\phi}^{6}\right)^{n_{3}} \cdots
$$

где $\widehat{\phi}$ - оператор в $L^{2}\left(\mathbb{R}^{d}\right)$ с ядром $\phi(x, y)$. Далее, $\operatorname{Tr} \widehat{\phi}^{2 n} \leqslant\|\widehat{\phi}\|^{n}\|\phi\|^{n}$. Поэтому выражение (32) не превосходит

$$
b e^{-c n} \sum_{k=0}^{n}\left(a_{2}\|\phi\|\right)^{k} k !\|\widehat{\phi}\|^{k} \sum_{2 n_{2}+3 n_{3}+\cdots=k} \frac{1}{2^{n_{2}} n_{2} ! 3^{n_{3}} n_{3} ! \cdots} .
$$

Сумма по $n_{2}, n_{3}, \ldots$ не превосходит 1 , а $\|\widehat{\phi}\|=O\left(\hbar^{d / 2}\right)$. Отсюда получаем формулу (19). Лемма 3 доказана.

6. Оценка невязки. Для оценки невязки воспользуемся следующим вспомогательным утверждением. Переобозначим операторы рождения и уничтожения следующим образом:

$$
c_{1}^{ \pm}(x)=a^{ \pm}(x), \quad c_{2}^{ \pm}(x)=b^{ \pm}(x)
$$

Рассмотрим оператор в пространстве Фока:

$$
\begin{aligned}
\widehat{A}= & \int d x_{1} \cdots d x_{m} d y_{1} \cdots d y_{l} \sum_{i_{1}, \ldots, i_{m}, j_{1}, \ldots, j_{l}=1,2} A_{i_{1} \ldots i_{m} j_{1} \ldots j_{l}}\left(x_{1}, \ldots, x_{m} ; y_{1}, . ., y_{l}\right) \\
& \times c_{i_{1}}^{+}\left(x_{1}\right) \cdots c_{i_{m}}^{+}\left(x_{m}\right) c_{j_{1}}^{-}\left(y_{1}\right) \cdots c_{j_{l}}^{-}\left(y_{l}\right),
\end{aligned}
$$

содержащий $m$ операторов рождения и $l$ операторов уничтожения. Пусть $\mathscr{F} n-$ подпространство $\mathscr{F}$, состоящее из всех векторов пространства Фока, все компоненты которых, кроме компонент с номерами $(k, n-k)$, обращаются в 0 . Обозначим через $\widehat{A}^{(n, n+l-m)}$ оператор $\widehat{A}^{(n, n+l-m)}: \mathscr{F}_{n+l-m} \rightarrow \mathscr{F}_{n}$, совпадающий с оператором $\widehat{A}_{\text {на }} \mathscr{F}_{n+l-m}$. Пусть $A: L^{2}\left(\mathbb{R}^{d l}\right) \rightarrow L^{2}\left(\mathbb{R}^{d m}\right)$ - оператор с ядром $A_{i_{1} \ldots i_{m} j_{1} \ldots j_{l}}\left(x_{1}, \ldots, x_{m} ; y_{1}, \ldots, y_{l}\right)$.

Лемма 8. Для нормы оператора $\widehat{A}^{(n, n+l-m)}$ справедлива оценка

$$
\left\|\widehat{A}^{(n, n+l-m)}\right\| \leqslant(n+l)^{(l+m) / 2}\|A\| .
$$


ДокАЗАТЕльСтво. Для простоты обозначений введем произвольный ортонормированньй базис $\left\{\varphi_{s}, s=1,2, \ldots\right\}$ в $L^{2}\left(\mathbb{R}^{d l}\right)$; рассмотрим операторы

$$
C_{2 s+1}^{ \pm}=\int d x a^{ \pm}(x) \varphi_{s}(x), \quad C_{2 s}^{ \pm}=\int d x b^{ \pm}(x) \varphi_{s}(x) .
$$

Оператор $\widehat{A}$ может быть представлен в виде

$$
\widehat{A}=\sum_{I_{1}, \ldots, I_{m}, J_{1}, \ldots, J_{l}=1}^{\infty} C_{I_{1}}^{+} \cdots C_{I_{m}}^{+} C_{J_{1}}^{-} \cdots C_{J_{l}}^{-} A_{I_{1} \ldots I_{m} J_{1} \ldots J_{l}} .
$$

Пусть $\Phi \in \mathscr{F}_{n+l-m}$, т.е.

$$
\Phi=\frac{1}{\sqrt{(n+l-m) !}} \sum_{K_{1}, \ldots, K_{n+l-m}} \varphi_{K_{1} \ldots K_{n+l-m}} C_{K_{1}}^{+} \cdots C_{K_{n+l-m}}^{+} \Phi^{(0)} .
$$

Квадрат нормы $\Phi$ имеет вид

$$
\|\Phi\|^{2}=\sum_{K_{1}, \ldots, K_{n+l-m}}\left|\varphi_{K_{1} \ldots K_{n+l-m}}\right|^{2} .
$$

Из определения операторов рождения и уничтожения вытекает оценка для квадрата нормы $\widehat{A} \Phi$ :

$$
\begin{aligned}
\|\widehat{A} \Phi\|^{2} \leqslant & n(n-1) \cdots(n-m+1)(n-m+1) \cdots(n-m+l) \\
& \times \sum_{I_{1}, \ldots, I_{m}, K_{1}, \ldots, K_{n-m}}\left|\sum_{J_{1}, \ldots, J_{l}} A_{I_{1} \ldots I_{m} J_{1} \ldots J_{l}} \varphi_{J_{1} \ldots J_{l} K_{1} \ldots K_{n-m}}\right|^{2} .
\end{aligned}
$$

Из формул (30) вытекает, что

$$
\|\widehat{A} \Phi\| \leqslant(n+l)^{(m+l) / 2}\|A\|\|\Phi\| .
$$

Лемма 8 доказана.

Из леммы 8 вытекает следующее утверждение. Рассмотрим оператор вида

$$
\widehat{B}=(2 \pi \hbar)^{d}: \int d x d y A_{1}(x) C_{i}^{+}(y) B\left(x,-i \hbar \frac{\partial}{\partial x}, y,-i \hbar \frac{\partial}{\partial y}\right) C_{j}^{-}(y) A_{2}(x):
$$

где функция $B\left(x, p_{x}, y, p_{y}\right)$ принадлежит пространству Шварца, $i$ и $j$ пробегают значения 1,2 , а каждый из операторов $A_{1}(x)$ и $A_{2}(x)$ является одним из четырех операторов $a^{ \pm}(x), b^{ \pm}(x)$. Знак нормального упорядочивания означает, что операторы рождения расставлены слева от операторов уничтожения.

ЛЕмма 9. При $\xi \in \mathscr{F}_{n}$ и достаточно малых $\hbar$

$$
\|\widehat{B} \xi\| \leqslant b_{1}(n+3)^{2} \hbar^{d / 2}\|\xi\|
$$

для некоторой не зависящей от $\hbar$ и $п$ константы $b_{1}$. 
ДокАЗАтЕльство. Рассмотрим сначала случай, когда один из операторов $A_{1}, A_{2}$ является оператором рождения, а другой - оператором уничтожения. В этом случае согласно лемме 8 справедлива оценка

$$
\|\widehat{B} \xi\| \leqslant b_{2}(n+3)^{2}(2 \pi \hbar)^{d}\|\xi\|
$$

где через $b_{2}$ обозначена норма псевдодифференциального оператора

$$
B\left(x,-i \hbar \frac{\partial}{\partial x}, y,-i \hbar \frac{\partial}{\partial y}\right)
$$

с вейлевским символом из пространства Шварца. Покажем, что эта норма при достаточно малых $\hbar$ не превосходит $\max B+\delta$ для любого $\delta>0$. Достаточно показать, что оператор $\Gamma=(\max B+\delta)^{2}-B^{+} B$ является положительно определенньм. Решая уравнение

$$
\Gamma-\delta_{1}=\widehat{B}_{1}^{2}, \quad \text { где } \widehat{B}_{1}=\widehat{B}_{1}^{+}=B_{1}\left(x,-i \hbar \frac{\partial}{\partial x}, y,-i \hbar \frac{\partial}{\partial y}\right)
$$

относительно символа оператора $B_{1}$ по теории возмущений по $\hbar$ для некоторого $\delta_{1}>0$, получаем представление для $\Gamma$ в виде суммы константы $\delta_{1}$, неотрицательно определенного оператора $B_{1}^{+} B_{1}$ и оператора $\hbar^{d} B_{2}$, норма которого не превосходит $\hbar^{d}\left\|B_{2}\right\|_{2}=$ $O\left(\hbar^{d / 2}\right): \Gamma=\delta_{1}+B_{1}^{+} B_{1}+\hbar^{d} B_{2}$. Тем самым, $\Gamma>0$ и $\|B\|$ ограничена равномерно по $\hbar$.

Рассмотрим теперь случай, когда операторы $A_{1}$ и $A_{2}$ оба являются операторами рождения или уничтожения. Ввиду инвариантности нормы относительно эрмитова сопряжения достаточно рассмотреть один из этих случаев, например, случай операторов рождения. Для оценки нормы оператора $\widehat{B}^{(31)}: \mathscr{F}_{1} \rightarrow \mathscr{F}_{3}$ рассмотрим оператор $\left(\widehat{B}^{(31)}\right)^{+} \widehat{B}^{(31)}$, представляющийся в виде произведения $(2 \pi \hbar)^{d}$ на $\hbar$-псевдодифференциальньй оператор с регулярным при $\hbar \rightarrow 0$ вейлевским символом. Ввиду изложенного норма этого оператора порядка $O\left(\hbar^{d}\right)$. Отсюда вытекает утверждение леммы 9.

Перейдем теперь к подстановке вектора пространства Фока (8) в уравнение (5) и оценке невязки. Для этого воспользуемся правилами коммутации (7), а также соотношением

$$
\begin{aligned}
& U_{\widehat{\varphi}}^{-1} \frac{d}{d t} U_{\widehat{\varphi}}=\frac{1}{2} \operatorname{Tr}\left(\left(1-\sigma \hat{\varphi}^{+} \widehat{\varphi}\right)^{-1}\left(\dot{\hat{\varphi}}^{+} \widehat{\varphi}-\dot{\hat{\varphi}}^{+} \widehat{\varphi}\right)\right) \\
& +a^{+}\left(\left(1-\sigma \hat{\varphi} \hat{\varphi}^{+}\right)^{-1 / 2} \dot{\hat{\varphi}}\left(1-\sigma \hat{\varphi}^{+} \hat{\varphi}\right)^{-1 / 2}\right) b^{+} \\
& -b^{-}\left(\left(1-\sigma \hat{\varphi}^{+} \hat{\varphi}\right)^{-1 / 2} \dot{\hat{\varphi}}^{+}\left(1-\sigma \hat{\varphi} \hat{\varphi}^{+}\right)^{-1 / 2}\right) a^{-} \\
& +a^{+}\left(\left(1-\sigma \widehat{\varphi} \hat{\varphi}^{+}\right)^{-1 / 2}\left(\left(1-\sigma \widehat{\varphi} \hat{\varphi}^{+}\right)^{1 / 2}\right)\right. \text {. } \\
& \left.+\sigma\left(1-\sigma \widehat{\varphi} \hat{\varphi}^{+}\right)^{-1 / 2} \dot{\hat{\varphi}} \hat{\varphi}^{+}\left(1-\sigma \widehat{\varphi} \hat{\varphi}^{+}\right)^{-1 / 2}\right) a^{-} \\
& +b^{+}\left(\left(1-\sigma \widehat{\varphi}^{T} \widehat{\varphi}^{*}\right)^{-1 / 2}\left(\left(1-\sigma \widehat{\varphi}^{T} \widehat{\varphi}^{*}\right)^{1 / 2}\right)\right. \text {. } \\
& \left.+\sigma\left(1-\sigma \widehat{\varphi}^{T} \widehat{\varphi}^{*}\right)^{-1 / 2} \dot{\hat{\varphi}}^{T} \widehat{\varphi}^{*}\left(1-\sigma \widehat{\varphi}^{T} \hat{\varphi}^{*}\right)^{-1 / 2}\right) b^{-} \text {, }
\end{aligned}
$$

где точкой обозначена производная по $t$.

Применяя данное коммутационное соотношение, получаем следующее утверждение. Введем обозначения

$$
n_{1}=\widehat{\varphi}\left(1-\sigma \widehat{\varphi}^{+} \widehat{\varphi}\right)^{-1} \widehat{\varphi}^{+}, \quad n_{2}=\widehat{\varphi}^{+}\left(1-\sigma \widehat{\varphi} \widehat{\varphi}^{+}\right)^{-1} \widehat{\varphi}
$$




$$
\begin{gathered}
L^{(0)}=-\frac{i}{2} \operatorname{Tr}\left(\left(1-\sigma \widehat{\varphi}^{+} \widehat{\varphi}\right)^{-1}\left(\dot{\hat{\varphi}}^{+} \widehat{\varphi}-\dot{\hat{\varphi}}^{+} \widehat{\varphi}\right)\right)+H^{(0)} \\
H^{(0)}=\frac{1}{\hbar}\left(\frac{1}{(2 \pi \hbar)^{d}} \int d p d q\left(\frac{p^{2}}{2}+U(q)\right)\left(n_{1}(p, q)-n_{2}(p, q)\right)\right. \\
\left.+\frac{\varepsilon}{2(2 \pi \hbar)^{d}} \int d p d q d p^{\prime} d q^{\prime} V\left(q, q^{\prime}\right)\left(n_{1}(p, q) n_{1}\left(p^{\prime}, q^{\prime}\right)-n_{2}(p, q) n_{2}\left(p^{\prime}, q^{\prime}\right)\right)\right) \\
T_{1,2}=-\frac{\hbar^{2}}{2} \frac{\partial^{2}}{\partial x^{2}}+U(x)+\alpha \int V(x, \xi) n_{1,2}(p, \xi) d p d \xi \\
L^{(1)}=\frac{1}{\hbar}\left(a^{+}\left(1-\sigma \widehat{\varphi} \hat{\varphi}^{+}\right)^{-1 / 2}\left(T_{1} \widehat{\varphi}-\widehat{\varphi} T_{2}\right)\left(1-\sigma \widehat{\varphi}^{+} \widehat{\varphi}\right)^{-1 / 2} b^{+}\right) .
\end{gathered}
$$

ПРЕДЛОЖЕНИЕ. Справедливо соотношение

$$
\left(i \frac{d}{d t}-L\right) U_{\widehat{\varphi}^{t}} g^{t}=U_{\widehat{\varphi}^{t}}\left(-L^{(0)}-L^{(1)}-L^{(1)+}\right) g^{t}+O(1) .
$$

Таким образом, если числовая функция $L^{(0)}$ и оператор $L^{(1)}$ обрашаются в 0 , то невязка имеет порядок $O(1)$, а не $O(1 / \varepsilon)$. При $\widehat{\varphi}=\widehat{\varphi}^{+}$условие $L^{(0)}=0$ тривиально, а требование $L^{(1)}=0$ равносильно уравнению Хартри-Вигнера (6).

Приведем явньй вид слагаемого порядка $O(1)$ в формуле $(34)$. Обозначим

$$
H_{2}^{\prime}=\frac{1}{\hbar}\left(a^{+} \widetilde{T} a^{-}-b^{+} \widetilde{T} b^{-}\right)
$$

где

$$
\begin{aligned}
& \widetilde{T}=\left(1-\sigma \widehat{\varphi}^{2}\right)^{-1 / 2}\left(T_{1}-\sigma \widehat{\varphi} T_{1} \widehat{\varphi}\right)\left(1-\sigma \widehat{\varphi}^{2}\right)^{-1 / 2} \\
& \mathscr{A}^{-}(p, q)=\left(\frac{\hbar}{2 \pi}\right)^{d / 2} \int d \beta b^{-}\left(q-\frac{\hbar \beta}{2}\right) a^{-}\left(q-\frac{\hbar \beta}{2}\right) e^{-i \beta p}, \mathscr{A}^{+}(p, q)=\left(\mathscr{A}^{-}(p, q)\right)^{+}, \\
& H_{2}^{\prime \prime}=i \alpha\left(\frac{1}{2} \int d p d q d p^{\prime} d q^{\prime}\left(\mathscr{A}^{+}(p, q) \mathscr{A}^{+}\left(p^{\prime}, q^{\prime}\right)-\mathscr{A}^{-}(p, q) \mathscr{A}^{-}\left(p^{\prime}, q^{\prime}\right)\right)\right. \\
& \times\left(1-\sigma \varphi^{2}(p, q)\right)^{-1}\left(1-\sigma \varphi^{2}\left(p^{\prime}, q^{\prime}\right)\right)^{-1} \\
& \times\left(\varphi(p, q) \frac{\partial V\left(q, q^{\prime}\right)}{\partial q^{\prime}} \frac{\partial \varphi\left(p^{\prime}, q^{\prime}\right)}{\partial p^{\prime}}+\varphi\left(p^{\prime}, q^{\prime}\right) \frac{\partial V\left(q, q^{\prime}\right)}{\partial q} \frac{\partial \varphi(p, q)}{\partial p}\right) \\
&+ \int d p^{\prime} d q^{\prime} d p d q \mathscr{A}^{+}(p, q) \mathscr{A}^{-}\left(p^{\prime}, q^{\prime}\right)\left(1-\sigma \varphi^{2}(p, q)\right)^{-1}\left(1-\sigma \varphi^{2}\left(p^{\prime}, q^{\prime}\right)\right)^{-1} \\
&\left.\times\left(\varphi\left(p^{\prime}, q^{\prime}\right) \frac{\partial V\left(q, q^{\prime}\right)}{\partial q} \frac{\partial \varphi(p, q)}{\partial p}-\varphi(p, q) \frac{\partial V\left(q, q^{\prime}\right)}{\partial q^{\prime}} \frac{\partial \varphi\left(p^{\prime}, q^{\prime}\right)}{\partial p^{\prime}}\right)\right)
\end{aligned}
$$

Обозначим $H_{2}=H_{2}^{\prime}+H_{2}^{\prime \prime}$.

Лемма 10. Пусть оператор $\widehat{\varphi}=\widehat{\varphi}^{+}$удовлетворяет уравнению (9), а компоненты вектора $g^{t}$ с номерами, больиими $1 / \hbar^{\delta}, 0<\delta<1 / 8$, обращаются в 0 . Tогда

$$
\left\|\left(i \frac{d}{d t}-L\right) U_{\widehat{\varphi}^{t}} g^{t}-U_{\widehat{\varphi}^{t}}\left(i \frac{d}{d t}-H_{2}\right) g^{t}\right\| \rightarrow 0, \quad \hbar \rightarrow 0, \quad \varepsilon=\alpha(2 \pi \hbar)^{d} .
$$


Эта лемма доказывается с помощью правил коммутации (7), унитарности оператора $U_{\widehat{\varphi}^{t}}$ и лемм 8,9 .

Таким образом, при

$$
\left\|\left(i \frac{d}{d t}-H_{2}\right) g^{t}\right\| \rightarrow 0, \quad \hbar \rightarrow 0,
$$

вектор пространства $\Phi$ ока $U_{\widehat{\varphi}^{t}} g^{t}$ удовлетворяет приближенно при $\hbar \rightarrow 0$ уравнению $(5)$. С помощью процедуры оценки обратного оператора [11] получаем, что

$$
\left\|\Phi^{t}-U_{\widehat{\varphi}^{t}} g^{t}\right\|=O\left(\hbar^{d / 2-\delta_{2}}\right)
$$

для некоторого $0<\delta_{2}<d / 2$, где $\Phi^{t}$ - решение уравнения (5), удовлетворяюшее начальному условию $U_{\widehat{\varphi}^{0}} g^{0}$. Для доказательства теоремы 1 достаточно воспользоваться утверждением, которое доказьвается подстановкой вектора $g^{t}$ в формулу (35) и использованием лемм 3,8 .

ЛЕмма 11. Для вектора

$$
\begin{gathered}
g^{t}=\left(\operatorname{det}\left(1-\left(M^{t}\right)^{+} M^{t}\right)\right)^{1 / 4} \sum_{n=0}^{n_{0}(\hbar)} \frac{1}{n !}\left(\frac{(2 \pi \hbar)^{d}}{2} \int d x d y a^{+}(x) a^{+}(y)\right. \\
\left.\times M^{t}\left(-i \hbar \frac{\partial}{\partial x}, x,-i \hbar \frac{\partial}{\partial y}, y\right) b^{+}(y) b^{+}(x)\right)^{n} \Phi^{(0)}
\end{gathered}
$$

справедливо свойство (35).

Поскольку норма $N$-й компоненты вектора пространства $Ф$ ока не превосходит нормы самого вектора, для доказательства теоремы 2 достаточно проверить, что норма $\left\|\Phi_{N}^{t}\right\|$ превосходит $\hbar^{d / 2-\delta_{2}-\delta_{3}}$ для $0<\delta_{3}<d / 2-\delta_{2}$. Тем самым, утверждение теоремы 3 вытекает из следующей леммы.

ЛЕмма 12. При

$$
N=\frac{1}{(2 \pi \hbar)^{d}} \int d p d q n(p, q)+O(1)
$$

для некоторой константы $b_{3}$ справедлива оценка

$$
\left\|P_{N} U_{\widehat{\varphi}} g\right\| \geqslant b_{3} \hbar^{d / 4}
$$

ДокАЗАТЕЛЬСТво. В силу неравенства Коши-Буняковского

$$
\left\|P_{N} U_{\widehat{\varphi}} g\right\| \geqslant \frac{\left|\left(P_{N} U_{\widehat{\varphi}} \Phi^{(0)}, P_{N} U_{\widehat{\varphi}} g\right)\right|}{\left\|P_{N} U_{\widehat{\varphi}} \Phi^{(0)}\right\|} .
$$

Для доказательства леммы достаточно проверить, что при $\hbar \rightarrow 0$ скалярное произведение

$$
\sqrt{N}\left(P_{N} U_{\widehat{\varphi}} \Phi^{(0)}, P_{N} U_{\widehat{\varphi}} g\right)
$$

имеет ненулевой предел в случаях, когда $g$ имеет вид (36) и когда $g$-вакуумный вектор.

Скалярное произведение (37) представляется в виде

$$
\int_{-\pi / \sqrt{N}}^{\pi / \sqrt{N}} \frac{d \beta}{2 \pi}\left(U_{\widehat{\varphi}}^{+} \exp \left(-i \frac{\beta}{\sqrt{N}}\left(\int d x a^{+}(x) a^{-}(x)-N\right)\right) U_{\widehat{\varphi}} \Phi^{(0)}, g\right) .
$$


Найдем предел интеграла $(38)$ при $\hbar \rightarrow 0$. Сначала докажем, что модуль подьнтегрального выражения ограничен сверху равномерно по $N$ интегрируемой функцией переменной $\beta$. Отсюда по теореме Лебега будет следовать, что интеграл (38) стремится к интегралу от предела подьнтегрального выражения при $\hbar \rightarrow 0$.

Вектор

$$
y(\beta)=U_{\widehat{\varphi}}^{+} \exp \left(-i \frac{\beta}{\sqrt{N}}\left(\int d x a^{+}(x) a^{-}(x)-N\right)\right) U_{\widehat{\varphi}} \Phi^{(0)}
$$

удовлетворяет уравнению

$$
i \sqrt{N} \frac{d y}{d \beta}=U_{\widehat{\varphi}}^{+}\left(\int d x a^{+}(x) a^{-}(x)-N\right) U_{\widehat{\varphi}} y
$$

и начальному условию $y(0)=\Phi^{(0)}$. Решение этой задачи Коши имеет вид

$$
y(\beta)=c_{1} \exp \left(\int d x a^{+}(x)\left(\widehat{\alpha} b^{+}\right)(x)\right) \Phi^{(0)},
$$

где

$$
\begin{gathered}
\alpha(\beta)=\widehat{\varphi}\left(e^{-i \beta / \sqrt{N}}-1\right)\left(1-\sigma \widehat{\varphi}^{2} e^{-i \beta / \sqrt{N}}\right)^{-1}, \\
c_{1}=\left(\operatorname{det}\left(1-\sigma \widehat{\varphi}^{2}\right)\left(1-\sigma \widehat{\varphi}^{2} e^{-i \beta / \sqrt{N}}\right)^{-1}\right)^{\sigma} e^{i \beta \sqrt{N}} .
\end{gathered}
$$

В силу неравенства Коши-Буняковского

$$
|(y(\beta), g)| \leqslant \sum_{n=0}^{n_{0}(\hbar)}\left\|y_{n}(\beta)\right\|\left\|g_{n}\right\|
$$

Для $\left\|g_{n}\right\|$ согласно лемме 3 справедлива оценка $\left\|g_{n}\right\| \leqslant b e^{-c n} /\left(1-a n_{0} \hbar^{d / 2}\right)$, т.е. $\left\|g_{n}\right\| \leqslant$ $b_{1} e^{-c n / 2}$ для некоторой константы $b_{1}$. Аналогично формуле $(32)$ получаем следующую оценку для квадрата нормы $n$-й компоненты $y_{n}$ :

$$
\left\|y_{n}\right\|^{2} \leqslant c_{2} \sum_{k=0}^{n} \frac{1}{(n-k) !}\|\widehat{\alpha}\|_{2}^{2 n-k}\|\widehat{\alpha}\|^{k} e^{-\|\widehat{\alpha}\|_{2}^{2}}
$$

для некоторой не зависяшей от $\beta$ константы $c_{2}$. Максимум суммы (39) достигается при $k=0$. Таким образом, имеет место оценка

$$
|(y(\beta), g)| \leqslant \sum_{n=0}^{n_{0}(\hbar)} b_{2} \frac{\sqrt{n+1}}{\sqrt{n !}}\|\widehat{\alpha}\|_{2}^{n} e^{-\|\widehat{\alpha}\|_{2}^{2}} e^{-c n / 2} .
$$

Tak кak

$$
\sum_{n=1}^{\infty} \frac{\xi^{n}}{\sqrt{(n-1) !}} \leqslant(1+\xi)\left(1+\xi^{2} e^{\xi^{2} / 2}\right), \quad \xi>0,
$$

и $\|\alpha\|_{2} \geqslant c_{4} \beta$, выражение (40) ограничено равномерно по $\hbar$ интегрируемой функцией $c_{5} e^{-c_{6} \beta^{2}}, c_{5}, c_{6}>0$. 
Найдем предел подьнтегрального выражения в формуле (38) при $\beta=$ const. В силу равномерной ограниченности величины $\sum_{n}\left|\left(y_{n}(\beta), g_{n}\right)\right|$ под знаком суммы $\sum_{n}\left(y_{n}(\beta)\right.$, $\left.g_{n}\right)$ можно перейти к пределу $\hbar \rightarrow 0$.

Обозначим через $\mathscr{F}_{p q}$ пространство Фока, состоящее из наборов функций $\left\{f_{n}\left(p_{1}, q_{1}\right.\right.$, $\left.\left.\ldots, p_{n}, q_{n}\right)\right\}$, симметричных относительно перестановок пар $\left(p_{i}, q_{i}\right) \in \mathbb{R}^{2 d}$. Введем в нем операторы рождения и уничтожения $A^{ \pm}(p, q)$. Обозначим через $\widetilde{\varphi}^{(0)}$ вакуумньй вектор этого пространства,

$$
\begin{gathered}
\widetilde{y}(\beta)=\exp \left(-\frac{\beta^{2}}{2} \sqrt{\int d p d q|\rho(p, q)|^{2}}+\beta \int d p d q A^{+}(p, q) \rho(p, q)\right) \widetilde{\varphi}^{(0)}, \\
\widetilde{g}=\left(\operatorname{det}\left(1-M^{+} M\right)\right)^{1 / 4} \exp \left(\frac{1}{2} \int d p d q d p^{\prime} d q^{\prime} A^{+}(p, q) M\left(p, q, p^{\prime}, q^{\prime}\right) A^{+}\left(p^{\prime}, q^{\prime}\right)\right) \tilde{\varphi}^{(0)},
\end{gathered}
$$

где

$$
\rho(p, q)=i \varphi(p, q)\left(1-\sigma \varphi^{2}(p, q)\right)\left(\int n(p, q) d p d q\right)^{-1} .
$$

При $n=$ const, $\hbar \rightarrow 0$ из леммы 4 вытекает, что

$$
\left(y_{n}(\beta), g_{n}\right) \rightarrow\left(\widetilde{y}_{n}(\beta), \widetilde{g}_{n}\right), \quad \hbar \rightarrow 0,
$$

так как слагаемые с $k \neq 0$ в формуле (23) дают стремящийся к 0 вклад в сумму. Из формулы (41) следует, что

$$
(y(\beta), g) \rightarrow(\widetilde{y}(\beta), \widetilde{g}), \quad \hbar \rightarrow 0 .
$$

Поэтому при $\hbar \rightarrow 0$ интеграл (38) стремится к

$$
\int_{-\infty}^{+\infty} \frac{d \beta}{2 \pi}(\widetilde{y}(\beta), g) \neq 0
$$

Лемма 12 доказана.

7. Геометрическая интерпретация асимптотических формул. Оказьвается, что полученные уравнения (9) и (11), через решения которых выражается асимптотическая формула, могут быть проинтерпретированы как система Гамильтона и уравнение эволюции комплексного ростка соответственно. При этом симплектическая структура фазового пространства оказьвается нетривиальной.

Обозначим через $\mathscr{M}$ пространство функций $\varphi(p, q)$ на $\mathbb{R}^{2 d}$ со значениями в множестве $\widetilde{\mathscr{M}}$. В бозевском случае множество $\widetilde{\mathscr{M}}$ выбирается в виде единичного круга на комплексной плоскости, в фермиевском - в виде римановой сферы. Будем отождествлять касательные векторы к многообразию в несингулярных точках с комплексньми функциями $\delta \varphi(p, q)$.

Рассмотрим 1-форму действия

$$
\omega_{1}[\delta \varphi]=\frac{i}{2} \int d p d q\left(\left(1-\sigma \widehat{\varphi}^{+} \widehat{\varphi}\right)^{-1}\left(\widehat{\varphi}^{+} \widehat{\delta \varphi}-\widehat{\delta \varphi}^{+} \widehat{\varphi}\right)\right)(p, q),
$$

где через $\widehat{\varphi}$ обозначен оператор с вейлевским символом $\varphi(p, q)$; аналогичное обозначение принято и для $\widehat{\delta \varphi}$. 
Дифференциал 1-формы (42) имеет вид $\omega_{2}=d \omega_{1}$,

$$
\begin{aligned}
\omega_{2}\left[\delta \varphi_{1}, \delta \varphi_{2}\right]= & \frac{i}{2} \int d p d q\left(\widehat{\delta \varphi}_{1}^{+}\left(1-\sigma \widehat{\varphi} \widehat{\varphi}^{+}\right)^{-1} \widehat{\delta \varphi}_{2}\left(1-\sigma \widehat{\varphi}^{+} \widehat{\varphi}\right)^{-1}\right. \\
& \left.-\widehat{\delta \varphi}\left(1-\sigma \hat{\varphi}^{+} \widehat{\varphi}\right)^{-1} \widehat{\delta \varphi}_{2}^{+}\left(1-\sigma \widehat{\varphi} \widehat{\varphi}^{+}\right)^{-1}\right)(p, q) .
\end{aligned}
$$

При $\hbar \rightarrow 0$ подынтегральное выражение в (43) переходит в элемент площади римановой сферы (в ферми-случае) либо плоскости Лобачевского, представленной в виде единичного круга (в бозевском случае). Отметим, что фазовые пространства в виде сферы и плоскости Лобачевского рассматривались в [12].

Лемма 13. Классическое уравнение движения

$$
i \dot{\hat{\varphi}}=T_{1} \widehat{\varphi}-\widehat{\varphi} T_{1}
$$

является гамильтоновой системой для рассматриваемого фазового пространства, если функиия Гамильтона $H^{(0)}\left(\varphi^{*}, \varphi\right)$ имеет вид (33).

ЗАмЕчание 1 . При $\widehat{\varphi}^{+}=\widehat{\varphi}$ уравнение (44) переходит в (9).

ЗАмЕчАниЕ 2 . При $\hbar \rightarrow 0$ формула (33) для гамильтонианапереходит в следующую:

$$
\begin{aligned}
H^{(0)}\left(\varphi^{*}, \varphi\right)= & i \int d p d q\left(\frac{p^{2}}{2}+U(q)+\alpha \int V\left(q, q^{\prime}\right) \frac{\left|\varphi\left(p^{\prime}, q^{\prime}\right)\right|^{2}}{1-\sigma\left|\varphi\left(p^{\prime}, q^{\prime}\right)\right|^{2}} d p^{\prime} d q^{\prime}\right) \\
& \times \frac{1}{\left(1-\sigma\left|\varphi\left(p^{\prime}, q^{\prime}\right)\right|^{2}\right)^{2}}\left(\frac{\partial \varphi}{\partial q} \frac{\partial \varphi^{*}}{\partial p}-\frac{\partial \varphi^{*}}{\partial p} \frac{\partial \varphi}{\partial q}\right) .
\end{aligned}
$$

ЗАМЕчАнИЕ 3 . Заменой

$$
n=\frac{|\varphi|^{2}}{1-\sigma|\varphi|^{2}}, \quad \alpha=\frac{1}{i} \ln \frac{\varphi}{|\varphi|}
$$

гамильтониан $H^{(0)}$ и 1-форма $\omega_{1}$ приводятся к не зависящему от типа статистики виду

$$
\begin{gathered}
\omega_{1}=\int d p d q \alpha(p, q) \delta n(p, q) \\
H^{(0)}=\int d p d q\left(\frac{p^{2}}{2}+U(q)+\alpha \int d p^{\prime} d q^{\prime} V\left(q, q^{\prime}\right) n\left(p^{\prime}, q^{\prime}\right)\left(\frac{\partial n}{\partial q} \frac{\partial \alpha}{\partial p}-\frac{\partial n}{\partial p} \frac{\partial \alpha}{\partial q}\right)\right) .
\end{gathered}
$$

В ферми-случае возникает ограничение на одночастичную плотность: $n(p, q)<1$.

Исследуем связь уравнения (11) с понятием комплексного ростка. Отождествим комплексифицированное касательное пространство $T \mathscr{M}_{\varphi}^{\mathbb{C}}$ к многообразию $\mathscr{M}$ в точке $\varphi$ с пространством $L^{2}\left(\mathbb{R}^{2 d}\right) \times L^{2}\left(\mathbb{R}^{2 d}\right)$ упорядоченных пар независимых комплексных функций $(\delta \varphi, \overline{\delta \varphi})$. При $\hbar \rightarrow 0$ комплексифицированная 2-форма $\omega_{2}^{\mathbb{C}}$ принимает вид

$$
\omega_{2}^{\mathbb{C}}\left(\left(\delta \varphi_{1}, \overline{\delta \varphi_{1}}\right),\left(\delta \varphi_{2}, \overline{\delta \varphi_{2}}\right)\right)=\frac{i}{2} \int \frac{d p d q}{\left(1-\sigma \varphi^{*} \varphi\right)^{2}}\left(\overline{\delta \varphi_{1}} \delta \varphi_{2}-\overline{\delta \varphi_{2}} \delta \varphi_{1}\right)
$$

Введем в $T \mathscr{M}_{\varphi}^{\mathbb{C}}$ инволюцию $(\delta \varphi, \overline{\delta \varphi})^{*}=(\overline{\delta \varphi}, \delta \varphi)$. 
ОПРЕДЕЛЕНИЕ 2. Пусть $M$ - оператор Гильберта-Шмидта в пространстве $L^{2}\left(\mathbb{R}^{2 d}\right)$ с симметричньм ядром и нормой меньше 1. Подпространство $\mathscr{G}_{M} \in T \mathscr{M}_{\varphi}^{\mathbb{C}}$ вида

$$
\mathscr{G}_{M}=\left\{\left(M\left(1-\sigma \varphi^{*} \varphi\right) \zeta,\left(1-\sigma \varphi^{*} \varphi\right) \zeta\right) \mid \zeta \in L^{2}\left(\mathbb{R}^{2 d}\right)\right\}
$$

назьвается комплексным ростком, отвечающим оператору $M$.

ЛЕмма 14. Комплексный росток удовлетворяет следующим свойствам:

1) $n p u \xi, \eta \in \mathscr{G}_{M} \omega_{2}^{\mathbb{C}}(\xi, \eta)=0$;

2) $n p u \xi \in \mathscr{G}_{M}, \xi \neq 0, i^{-1} \omega_{2}^{\mathbb{C}}\left(\xi, \xi^{*}\right)>0$;

3) упорядоченная пара функиий $(\delta \varphi, \overline{\delta \varphi})$ принадлежсит ростку $\mathscr{G}_{M^{t}}$ тогда и только тогда, когда является решением задачи Коши комплексифицированной системы в вариачиях для системы Гамильтона (27) с начальным условием на ростке $\mathscr{G}_{M^{0}}$.

Свойства 1) и 2) вытекают из определения 2, свойство 3) является следствием уравнения (11). Доказательство этих свойств аналогично [4], [7].

\section{СПИСОК ЦИТИРОВАННОЙ ЛИТЕРАТУРЫ}

[1] Maslov V.P., Shvedov O. Yu. Asymptotic solutions to the Wigner equation for systems of a large number of particles // Russian J. Math. Phys. 1995. V. 3. № 1. P. 65-80.

[2] Маслов В. П. Комплексный метод ВКБ в нелинейных уравнениях. М.: Наука, 1977.

[3] Маслов В. П., Шведов О. Ю. Метод комплексного росткав пространстве Фока. І. Асимптотики типа волновых пакетов // ТМФ. 1995. Т. 104. № 2. С. 310-329.

[4] Маслов В.П., Шведов О.Ю. Метод комплексного ростка в пространстве Фока. II. Асимптотики, отвечающие конечномерным изотропным многообразиям // ТМФ. 1995. Т. 104. № 3. C. $479-506$.

[5] Березин Ф. А. Метод вторичного квантования. М.: Наука, 1986.

[6] Карасёв М. В., Маслов В. П. Нелинейная скобка Пуассона. Геометрия и квантование. М.: Наука, 1991.

[7] Maslov V.P., Shvedov O. Yu. An asymptotic formula for the $N$-particle density function as $N \rightarrow \infty$ and violation of the chaos hypothesis // Russian J. Math. Phys. 1994. V. 2. № 2. P. 217-234.

[8] Maslov V.P., Shvedov O. Yu. On the approximation for the quantum large canonical distribution // Russian J. Math. Phys. 1998. V. 6. № 1. P. 80-89.

[9] Кострикин А. И. Введение в алгебру. М.: Наука, 1977.

[10] Маслов В. П. Операторные методы. М.: Наука, 1973.

[11] Маслов В. П. Теория возмущений и асимптотические методы. М.: Изд-во МГУ, 1965.

[12] Berezin F. A. Models of Gross-Neveu type are quantization of a classical mechanics with nonlinear phase space // Comm. Math. Phys. 1978. V. 63. P. 131-153. 